\title{
Differential Privacy for Directional Data
}

\author{
Benjamin Weggenmann \\ SAP Security Research \\ Karlsruhe, Germany
}

\begin{abstract}
Directional data is an important class of data where the magnitudes of the data points are negligible. It naturally occurs in many realworld scenarios: For instance, geographic locations (approximately) lie on a sphere, and periodic data such as time of day, or day of week can be interpreted as points on a circle. Massive amounts of directional data are collected by location-based service platforms such as Google Maps or Foursquare, who depend on mobility data from users' smartphones or wearable devices to enable their analytics and marketing businesses. However, such data is often highly privacysensitive and hence demands measures to protect the privacy of the individuals whose data is collected and processed. Starting with the von Mises-Fisher distribution, we therefore propose and analyze two novel privacy mechanisms for directional data by combining directional statistics with differential privacy, which presents the current state-of-the-art for quantifying and limiting information disclosure about individuals. As we will see, our specialized privacy mechanisms achieve a better privacy-utility trade-off than ex post adaptions of established mechanisms to directional data.
\end{abstract}

\section{CCS CONCEPTS}

- Security and privacy $\rightarrow$ Data anonymization and sanitization; Pseudonymity, anonymity and untraceability; • Information systems $\rightarrow$ Spatial-temporal systems; Location based services.

\section{KEYWORDS}

directional data; differential privacy; data anonymization

\section{ACM Reference Format:}

Benjamin Weggenmann and Florian Kerschbaum. 2021. Differential Privacy for Directional Data. In Proceedings of the 2021 ACM SIGSAC Conference on Computer and Communications Security (CCS '21), November 15-19, 2021, Virtual Event, Republic of Korea. ACM, New York, NY, USA, 18 pages. https: //doi.org/10.1145/3460120.3484734

\section{INTRODUCTION}

In recent years, large-scale collection and processing of directional data have become important drivers for the digital economy: For instance, crowd-sourced data from mobile or wearable devices often includes the geographic location where and the time when the data was recorded. Prominent applications include location-based marketing and analytics, as provided by platforms such as Foursquare, and the collection of check-in data by online mapping services such

Permission to make digital or hard copies of part or all of this work for personal or classroom use is granted without fee provided that copies are not made or distributed for profit or commercial advantage and that copies bear this notice and the full citation on the first page. Copyrights for third-party components of this work must be honored. For all other uses, contact the owner/author(s).

CCS '21, November 15-19, 2021, Virtual Event, Republic of Korea

(C) 2021 Copyright held by the owner/author(s)

ACM ISBN 978-1-4503-8454-4/21/11.

https://doi.org/10.1145/3460120.3484734

\author{
Florian Kerschbaum \\ University of Waterloo \\ Waterloo, ON, Canada
}

as Google Maps who provide, e.g., daily "busyness" histograms of visit times at places like stores or restaurants, from which users can estimate how busy a location is during different times of the day.

While such techniques provide substantial value for businesses and drive innovation, the data collected in such scenarios is often privacy-sensitive, and users may be reluctant to share their whereabouts during the course of the day. In many cases, directional data conveys particularly sensitive information, as illustrated by recent news about location tracking on smartphones or fitness trackers $[18,37]$. Personal locations are suspect to various attacks, $\mathrm{cf}$. the survey by Krumm [24], in particular when combined with temporal information as shown by Primault et al. [33] or Pyrgelis et al. [35].

Problem. To protect the privacy of individuals while maintaining data-driven business models, the concept of differential privacy (DP) by Dwork et al. [10] presents the current state-of-the-art for quantifying and limiting information disclosure about individuals. DP mechanisms have been proposed for various settings and data types, e.g., the standard Laplace mechanism [10] which extends infinitely on the real line, or the Planar Laplace mechanism by Andrés et al. [2] which is defined for planar locations. While post-processing, such as clipping or wrapping, can be applied to adapt these mechanisms to periodic domains, none of them intrinsically considers the potentially directional nature of the underlying data. In fact, adapted standard mechanisms based on wrapping can behave even worse than uniform noise, as we show in Section 4.2.1. We hence argue that specialized, directional privacy mechanisms are needed to provide superior privacy-utility trade-offs and investigate proper ways to provide DP intrinsically for directional data (cf. Section 3).

Inspired by the notion of geo-indistinguishability [2], a variant of metric privacy [4] for planar location data, we propose directional privacy as adaptation for directional data. As benefit, this notion allows relaxing the guarantees of pure DP to protect data within a given protection radius (i.e., surface distance or angle) $r>0$ with a specified privacy level $\ell$. By setting the protection radius $r=\Delta$ to the sensitivity, this also covers pure $\epsilon$-DP. Relaxing the privacy guarantees to a smaller radius is very useful when working in the local model, e.g., when we want to protect spatial or temporal data that are close to each other, such as restaurants or other venues in densely populated areas, where pure DP would inject too much noise. We demonstrate this in our experiments in Section 4.3.2.

As we observe in Section 4.2, several directional statistics such as the circular mean benefit from our specialized mechanisms: At $\epsilon=1.0$, we achieve a more than 4.8 -fold reduction in the number of required survey responses over adapted baselines to reach an error below 0.1 , so that the service provider needs to collect only $\approx 750$ responses instead of over 3600 . Conversely, given the same number of responses, our proposed mechanisms achieve MAEs of only 0.407 and 0.321 , which is less than half of 0.695 as for the Wrapped Laplace baseline. Strikingly, for such directional statistics, local DP is necessarily more accurate than central DP and hence 
the method of choice, since it does not require a trusted aggregator. Moreover, in Section 4.3, we observe that a wrapped Planar Laplace variant for geolocations yields larger errors for histograms than our proposed mechanisms in the critical range $10^{-1} \lesssim \epsilon \lesssim 10$.

Contributions. Our results concern theoretical aspects (Section 3) in the areas of privacy and directional statistics, as well as experiments (Section 4) to substantiate the theory and its applicability:

- As for privacy, in Section 3, we propose the notion of $d i-$ rectional privacy, an adaptation of metric privacy $[2,4]$ for directional data based on the surface distance on the sphere. To realize this notion, we form the novel von Mises-Fisher and Purkayastha privacy mechanisms from the eponymous distributions and prove their (differential) privacy properties.

- We derive analytical formula in terms of confluent hypergeometric series for the expected Euclidean distance and the cumulative distribution function (CDF) of the mixture density of the Von Mises-Fisher distribution in Section 3.2, as well as closed-form solutions in terms of elementary functions for the expected surface distance and the CDF of the angular density of the Purkayastha distribution in Section 3.3. We use those formulas to compare our directional with traditional baseline mechanisms in Section 3.6 and assess their error.

- In Section 3.5, we make use of our closed-form solution for the angular CDF to build an approximate inversion sampling method for the Purkayastha distribution. To our best knowledge, this is the first published method for this distribution which has been deemed numerically hard to sample from in dimensions over 150 [7]. Our benchmarks show that it is applicable in up to tens of thousands of dimensions.

- We apply our proposed mechanisms in several real-world settings and compare their privacy-utility trade-offs: We consider the periodic mean in the central and local privacy models for time-of-day data in Section 4.2, as well as histograms of location and time-of-day data in the local model in Section 4.3. We also illustrate privately collecting check-in time and location data to create "busyness" histograms of popular visit times even if the data curator is untrusted.

- Finally, we perform supplementary simulation experiments in Appendix B.1 to support the correctness of our derived formula for the expected distances and CDFs. Based on the empirical expected distances, we also compare the privacyutility trade-off for both mechanisms at a given privacy level.

\section{BACKGROUND}

This section provides terminology and results of differential privacy and directional statistics as required in the paper.

\subsection{Differential Privacy}

In the following, we introduce some basic notions of differential and metric privacy. We roughly adopt the notation of Chatzikokolakis et al. [4]. For a broader introduction and details, we refer the reader to the books by Dwork and Roth [11] or Li et al. [29].

Probability distributions. For a given set $\mathcal{Z}$, we denote by $\mathcal{P}_{\mathcal{Z}}$ the set of probability distributions (or measures) on $\mathcal{Z}$, i.e., the set of normed and $\sigma$-additive functions $\mathrm{P}: \sigma(\mathcal{Z}) \rightarrow[0,1]$ where $\sigma(\mathcal{Z})$ is a $\sigma$-algebra on $\mathcal{Z}$. The probability of an event $Z \in \sigma(\mathcal{Z})$ (i.e., a measurable subset of $\mathcal{Z}$ ) is thus given by $\mathrm{P}[\mathrm{Z}]$. A distribution $\mathrm{P}$ is typically specified by its probability density function (PDF), which we denote by $\mathrm{P}[\mathrm{z}]$ by slight reuse of notation. For univariate distributions on $\mathcal{Z} \subseteq \mathbb{R}$, we also denote the cumulative distribution function $(C D F)$ at $\mathrm{Z}$ by $\mathrm{P}[\mathrm{z} \leq \mathrm{Z}]$, shorthand for $\mathrm{P}[\{\mathrm{z} \in \mathbb{R}: \mathrm{z} \leq \mathrm{Z}\}]$.

We often consider families of distributions parametrized by one or more parameters, such as $\boldsymbol{\mu}$ or $\epsilon$, which we append in parentheses as in $\mathrm{P}(\boldsymbol{\mu}, \epsilon)[\cdot]$, or simply $\mathrm{P}(\boldsymbol{\mu}, \epsilon)$.

Definition 1. Let $\mathcal{X}$ and $\mathcal{Z}$ be two sets. A random mechanism from $\mathcal{X}$ to $\mathcal{Z}$ is a function $\mathcal{M}: \mathcal{X} \rightarrow \mathcal{P}_{\mathcal{Z}}$ that assigns to each input $x$ a probability distribution on $\mathcal{Z}$. $\mathcal{M}$ can be specified through a parametrized family of distributions $M(x)$ on $\mathcal{Z}$ via $\mathcal{M}(x)=M(x)$ for $x \in \mathcal{X}$; we then say $\mathcal{M}$ is the mechanism induced by $M$. From an algorithmic perspective, we run a random mechanism $\mathcal{M}$ on a given input $x$ by sampling a realization $\mathrm{z}$ from the distribution $\mathcal{M}(x)$. We write this as $\mathrm{z} \sim \mathcal{M}(x)$.

Differential privacy (DP) and its models. DP has been introduced by Dwork et al. [10] under the name $\epsilon$-indistinguishability. Its goal is to give semantic privacy by quantifying the risk of an individual that results from participation in data collection.

In the original, central model, we assume the collected data is stored in a central database with one record per participant. If we consider adjacent databases that differ by at most one record (i.e., one individual's data), a differentially private query on both databases should yield matching results with similar probabilities, i.e., answers that are probabilistically indistinguishable. This is achieved via random mechanisms on the universe of datasets $\mathcal{X}=\mathcal{D}$ that return noisy query results, thus masking the impact of each individual.

Definition 2. Let $\epsilon>0$ be a privacy parameter. A random mechanism $\mathcal{M}: \mathcal{D} \rightarrow \mathcal{P}_{\mathcal{Z}}$ fulfills $\epsilon-D P$ if for all adjacent databases $x, x^{\prime} \in \mathcal{D}$, and all sets of possible outputs $Z \subset \operatorname{supp} \mathcal{M}$,

$$
\mathcal{M}(x)[Z] \leq e^{\epsilon} \cdot \mathcal{M}\left(x^{\prime}\right)[Z] .
$$

In the local model [9], noise is added locally at the data source, before the data is collected and stored in a central database. A basic example is randomized response [43], where each survey participant either provides a truthful or a random answer depending on the flip of an (unbiased) coin.

2.1.1 Generalization with metrics. A limitation with DP is that the indistinguishability is achieved between two inputs on a per-record level regardless of their actual values. This can be especially problematic in the local model, where each user might just submit one single record, in which case a DP mechanism with small privacy parameter $\epsilon$ would enforce all submitted records to be indistinguishable, thus rendering the collected data essentially useless.

To the same end, Chatzikokolakis et al. [4] argue that in some scenarios, the (in)distinguishability between two databases as enforced by a privacy mechanism should depend on the values themselves instead of the number of differing records. They hence propose a generalized notion of privacy on metric spaces that also covers domains other than databases where a conforming mechanism run on nearby elements still has similar output probabilities: 
Definition 3 (Metric privacy). Let $\epsilon>0$ be a privacy parameter. On a metric space $(\mathcal{X}, d)$, a mechanism $\mathcal{M}$ satisfies $\epsilon d$-privacy if for all $x, x^{\prime} \in \mathcal{X}$ and all $\mathrm{Z} \subset \operatorname{supp} \mathcal{M}$,

$$
\mathcal{M}(x)[Z] \leq \exp \left(\epsilon \cdot d\left(x, x^{\prime}\right)\right) \cdot \mathcal{M}\left(x^{\prime}\right)[Z] .
$$

In other words, the level of indistinguishability of any two points $x, x^{\prime}$ is bounded by $\epsilon$ times their distance. Andrés et al. [2] provide another interpretation: If we consider an arbitrary but fixed distance $r>0$, any two points with $d\left(x, x^{\prime}\right) \leq r$ achieve a level of indistinguishability at most $\epsilon r$; hence, an $\epsilon d$-private mechanism $\mathcal{M}$ achieves a privacy level $\ell=\epsilon r$ within a protection radius $r$.

Note that we recover the original notion of $\epsilon$-DP on the space of databases $X=\mathcal{D}$ if we use the record-level edit distance $d_{ \pm 1}$, as datasets $x, x^{\prime} \in \mathcal{D}$ differ by at most one record if and only if $d_{ \pm 1}\left(x, x^{\prime}\right) \leq 1$. This motivates the following broader definition:

Definition 4. In a metric space $(\mathcal{X}, d)$, we say that two inputs $x, x^{\prime} \in \mathcal{X}$ are adjacent (with respect to $d$ ) if $d\left(x, x^{\prime}\right) \leq 1$. We write this as $x \sim{ }_{d} x^{\prime}$ (or $x \sim x^{\prime}$ if $d$ is understood from the context).

\subsection{Directional statistics}

Directional statistics is an area of statistics that is concerned with directions, i.e., data points whose magnitudes can be neglected. Hereafter, we introduce some terms and notions as required in the paper. As reference, we recommend the book by Mardia and Jupp [30].

Since directions are independent of magnitude, they can be identified by unit vectors, i.e., points on a unit sphere:

Definition 5. For $n \in \mathbb{N}$, the unit $(n-1)$-sphere

$$
\mathbb{S}^{n-1}:=\left\{x \in \mathbb{R}^{n}:\|x\|_{2}=1\right\}
$$

is the set of unit vectors in $n$-dimensional Euclidean space. We write $r \mathbb{S}^{n-1}$ for the $(n-1)$-sphere of radius $r>0$.

Fact 6. The surface area of the unit sphere $\mathbb{S}^{n-1}$ is given by its $(n-1)$-dimensional volume

$$
S_{n-1}:=\operatorname{vol}\left(\mathbb{S}^{n-1}\right)=2 \cdot \pi^{\frac{n}{2}} \cdot \Gamma^{-1}\left(\frac{n}{2}\right) .
$$

For a sphere of radius $r$, we have $\operatorname{vol}\left(r \mathbb{S}^{n-1}\right)=S_{n-1} r^{n-1}$.

Example 7. The uniform distribution Uni $\left(\mathbb{S}^{n-1}\right)$ on $\mathbb{S}^{n-1}$ has a constant PDF Uni $\left(\mathbb{S}^{n-1}\right)[\mathrm{x}] \equiv S_{n-1}^{-1}=\frac{1}{2} \Gamma\left(\frac{n}{2}\right) \pi^{-\frac{n}{2}}$.

2.2.1 Rotationally symmetric distributions. We consider unimodal distributions on $\mathbb{S}^{n-1}$ that are rotationally symmetric about the mode $\boldsymbol{\mu} \in \mathbb{S}^{n-1}$. The corresponding densities $\mathrm{P}[\mathrm{x}]$ depend on $\mathbf{x}$ only through the projection $t=\boldsymbol{\mu}^{\top} \mathbf{x}$ of $\mathbf{x}$ on the modal axis through $\boldsymbol{\mu}$, so all points $\mathbf{x}$ with $\boldsymbol{\mu}^{\top} \mathbf{x}=t$ have constant density $\mathrm{P}[\mathbf{x}]=\overline{\mathrm{P}}\left[\boldsymbol{\mu}^{\top} \mathbf{x}\right]=$ $\overline{\mathrm{P}}[t]$ for a corresponding kernel function $\overline{\mathrm{P}}:[-1,1] \rightarrow \mathbb{R}_{\geq 0}$.

To sample from such distributions, it is helpful to consider marginal distributions that are easier to handle. A way to obtain them is through the so-called tangent-normal decomposition (cf. Fig. 1): A random vector $\mathbf{x} \in \mathbb{S}^{n-1}$ can be decomposed into two components along the mode $\boldsymbol{\mu}$ and along a tangential unit vector $\xi \perp \boldsymbol{\mu}$ as

$$
\mathbf{x}=t \boldsymbol{\mu}+\sqrt{1-t^{2}} \xi, \quad \text { where } t=\boldsymbol{\mu}^{\top} \mathbf{x}
$$

Due to the rotational symmetry, $\xi \in \mathbb{S}^{n-2} \perp \boldsymbol{\mu}$ is distributed uniformly on the subsphere orthogonal to $\boldsymbol{\mu}$ (marked in green).

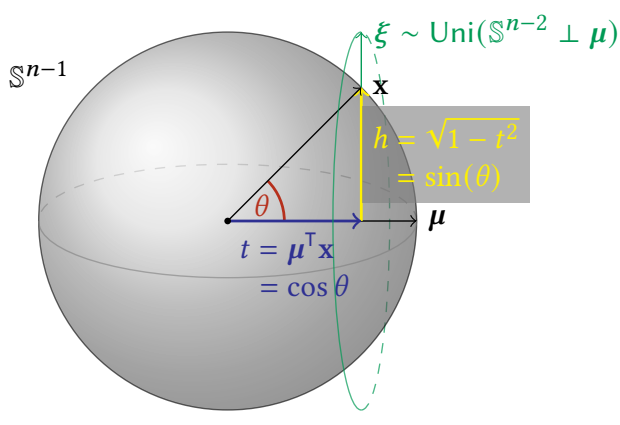

Figure 1: Tangent-normal decomposition of a random unit vector $\mathrm{x}$ into orthogonal components along the mode $\mu$ and a tangential vector $\xi \perp \boldsymbol{\mu}$ of lengths $t$ and $h$, respectively.

Following Ulrich [38], the length $t=\boldsymbol{\mu}^{\top} \mathbf{x}$ along $\boldsymbol{\mu}$ (marked in blue) is called the mixture variable. Its associated mixture density

$$
\operatorname{PMix}[t]=\int_{\mathbf{x}: \boldsymbol{\mu}^{\top} \mathbf{x}=t} \mathrm{P}[\mathbf{x}] \mathrm{dx}, \quad t \in[-1,1],
$$

can be evaluated as follows (proof in Appendix A.2):

Lemma 8 (Mixture density). Given a rotationally symmetric distribution $\mathrm{P}$ with kernel function $\overline{\mathrm{P}}[t]$, we can express its mixture density PMix $[t]$ in terms of the kernel function as

$$
\operatorname{PMix}[t]=S_{n-2} \cdot\left(1-t^{2}\right)^{\frac{n-3}{2}} \cdot \overline{\mathrm{P}}[t], \quad t \in[-1,1] .
$$

Alternatively, by substituting $t=\cos (\theta)$ in Eq. (1), we can write

$$
\mathbf{x}=\cos (\theta) \boldsymbol{\mu}+\sin (\theta) \boldsymbol{\xi},
$$

where $\theta=\arccos \left(\boldsymbol{\mu}^{\top} \mathbf{x}\right)$ is the angle or arc length between $\mathbf{x}$ and the mode $\boldsymbol{\mu}$ (marked in red). The angular density of $\theta$ is as follows:

Corollary 9 (Angular density). Given a rotationally symmetric distribution $\mathrm{P}$ with kernel function $\overline{\mathrm{P}}[t]$, we can express its angular density $\operatorname{PArc}[\theta]$ for an angle $\theta \in[0, \pi]$ as

$$
\operatorname{PArc}[\theta]=S_{n-2} \sin ^{n-2}(\theta) \cdot \overline{\mathrm{P}}[\cos (\theta)] .
$$

Importantly, the tangent-normal decomposition thus reduces the multivariate sampling problem $\mathbf{x} \sim \mathrm{P}$ to a univariate one, namely $t \sim$ PMix or $\theta \sim$ PArc, plus a uniform one, $\xi \sim \operatorname{Uni}\left(\mathbb{S}^{n-2} \perp \mu\right)$. This is normally easier, as generating univariate samples avoids the curse of dimensionality regarding numerical precision and complexity.

2.2.2 Von Mises-Fisher distribution. The $(n-1)$-dimensional von Mises-Fisher (VMF) distribution, named after von Mises [40] and Fisher [13], is a probability distribution on the unit hypersphere $\mathbb{S}^{n-1}$. Due to its popularity, it has been studied thoroughly, and proven sampling methods have been published [27, 38, 44] (see Section 3.4.1). Therefore, we use it as a starting point to construct a first novel privacy mechanism for directional data in Section 3.2.

Definition 10. The VMF distribution on $\mathbb{S}^{n-1}$ with mean direction $\boldsymbol{\mu} \in \mathbb{S}^{n-1}$ and concentration parameter $\kappa \geq 0$ is given by the density

$$
\operatorname{VMF}(\boldsymbol{\mu}, \kappa)[\mathbf{x}]=C_{\mathrm{VMF}}(n, \epsilon) \cdot \exp \left(\kappa \cdot \boldsymbol{\mu}^{\top} \mathbf{x}\right) .
$$

If we set $v:=\frac{n}{2}-1$, the normalization factor amounts to

$$
C_{\mathrm{VMF}}(n, \kappa)=\frac{\kappa^{v}}{(2 \pi)^{v+1} I_{v}(\kappa)}=\frac{\Gamma(v+1) e^{\kappa}}{2 \cdot \pi^{v+1} M\left(v+\frac{1}{2}, 2 v+1,2 \kappa\right)} .
$$


The parameter $\kappa$ characterizes how strongly the random vectors $\mathbf{x} \sim \operatorname{VMF}(\boldsymbol{\mu}, \kappa)$ are concentrated about the mean $\boldsymbol{\mu}$. If $\kappa>0$, the distribution is unimodal and the mode matches $\mu$. A VMF distribution with $\kappa=0$ degenerates to the uniform distribution Uni $\left(\mathbb{S}^{n-1}\right)$.

2.2.3 Purkayastha distribution. Purkayastha [34] studied rotationally symmetric distributions on $\mathbb{S}^{n-1}$ for which the median direction is a maximum likelihood estimate of the location parameter. $\mathrm{He}$ proposed the following distribution that meets this criterion; in Section 3.3, we use it for a second mechanism for directional data.

Definition 11. The Purkayastha distribution on $\mathbb{S}^{n-1}$ with mean direction $\boldsymbol{\mu} \in \mathbb{S}^{n-1}$ and concentration parameter $\kappa \geq 0$ has density

$$
\operatorname{Pur}(\boldsymbol{\mu}, \kappa)[\mathbf{x}]=C_{\text {Pur }}(n, \kappa) \cdot \exp \left(-\kappa \cdot \arccos \left(\boldsymbol{\mu}^{\top} \mathbf{x}\right)\right) .
$$

Its normalization factor is $C_{\mathrm{Pur}}(n, \kappa)=S_{n-2}^{-1} F_{n-2,-\kappa}^{-1}(\pi)$, where

$$
F_{n-2,-\kappa}^{-1}(\pi)= \begin{cases}\frac{\kappa\left(\kappa^{2}+2^{2}\right)\left(\kappa^{2}+4^{2}\right) \cdots\left(\kappa^{2}+(n-2)^{2}\right)}{(n-2) !\left(1-e^{-\kappa \pi}\right)} & \text { for even } n, \\ \frac{\left(\kappa^{2}+1^{2}\right)\left(\kappa^{2}+3^{2}\right) \cdots\left(\kappa^{2}+(n-2)^{2}\right)}{(n-2) !\left(1+e^{-\kappa \pi}\right)} & \text { for odd } n\end{cases}
$$

(cf. Lemma 21). Note that $F$ also appears in the normalization constant of the angular and mixture densities in Section 3.3.1.

\section{DIRECTIONAL PRIVACY MECHANISMS}

This section presents our main results. This comprises a novel notion of privacy for directional data as well as the conforming von Mises-Fisher and Purkayastha mechanisms. We derive certain marginal densities, expected values, and CDFs of the underlying distributions. These are important for assessing the average error, or sampling, as we show by constructing a novel Purkayastha sampling method. Moreover, we explain how the mechanism parameters depend on the desired privacy guarantees. Lastly, we describe adaptions of common privacy mechanisms to directional data as baselines. Proofs for this section are provided in Appendix A.3.

\subsection{Directional privacy}

Our goal is to define a variant of metric privacy [4] (Definition 3) for directions. To this end, we first need a suitable metric to measure distances between directions, i.e., angles on the sphere:

Definition 12. The surface distance between two points $x, y \in$ $r \mathbb{S}^{n-1}$ is given by the arc length $d_{\llcorner}(\boldsymbol{x}, \boldsymbol{y}):=r \arccos \left(\boldsymbol{x}^{\top} \boldsymbol{y}\right)$.

On the unit sphere $(r=1)$, the surface distance $d_{\measuredangle}$ between two points is the enclosed angle (in radians) between them-together, $\mathbb{S}^{n-1}$ with $d_{\llcorner}$becomes a metric space for angles. We thus obtain

Definition 13 (Directional privacy). Let $\epsilon>0$. A mechanism $\mathcal{M}$ on $\mathbb{S}^{n-1}$ fulfills $\epsilon d_{\llcorner}$-privacy if for all $x, x^{\prime} \in \mathbb{S}^{n-1}$ and all $\mathrm{Z} \subset \operatorname{supp} \mathcal{M}$,

$$
\mathcal{M}(x)[Z] \leq \exp \left(\epsilon \cdot d_{\llcorner}\left(x, x^{\prime}\right)\right) \cdot \mathcal{M}\left(x^{\prime}\right)[Z] .
$$

Interpretation as pure DP. Following Chatzikokolakis et al. [4, Fact 5], $\epsilon d$-privacy on a space $\mathcal{Y}$ implies $\epsilon \Delta$-DP for a query function $f: \mathcal{D} \rightarrow \mathcal{Y}$ with $d$-sensitivity $\Delta$ on the universe of databases $\mathcal{D}$. We apply this fact specifically to sphere-valued functions with range $y \subseteq \mathbb{S}^{n-1}$ to obtain $\epsilon$-DP:

Fact 14 ( $\epsilon d$-privacy implies $\epsilon$-DP). Let $f: \mathcal{D} \rightarrow \mathbb{S}^{n-1}$ be a query function, and let $\mathcal{M}_{\epsilon}$ be an $\epsilon d$-private mechanism on $\mathbb{S}^{n-1}$ with metric $d$. Then its $d$-sensitivity is $\Delta=\Delta_{d} f:=\max _{x \sim \mathcal{D}} y d(f(x), f(y))$, and the composition $\mathcal{M}_{\epsilon / \Delta} \circ f$ is $\epsilon$-differentially private.

\subsection{Von Mises-Fisher privacy mechanism}

The Laplace and Gaussian distributions are often used in Euclidean space, particularly as mechanisms to provide DP. Since the VMF distribution can be seen as natural counterpart on the sphere, we propose it as promising candidate to achieve DP for directional data:

Theorem 15 ( $\epsilon d_{2}$-privacy of VMF mechanism). Let $\epsilon>0$ be a privacy parameter. The VMF mechanism on $\mathbb{S}^{n-1}$ induced by $\boldsymbol{x} \mapsto$ $\operatorname{VMF}(x, \epsilon)$ for $x \in \mathbb{S}^{n-1}$ fulfills $\epsilon d_{2}$-privacy.

Corollary 16 ( $\epsilon d_{L}$-privacy of VMF mechanism). For any $x, y \in$ $\mathbb{S}^{n-1}, d_{2}(\boldsymbol{x}, \boldsymbol{y}) \leq d_{\llcorner}(\boldsymbol{x}, \boldsymbol{y})$, so the VMF mechanism fulfills $\epsilon d_{\llcorner}$-privacy.

By Fact 14, the VMF mechanism $\operatorname{VMF}(x, \epsilon / \Delta)$ also provides $\epsilon$ DP for sphere-valued functions $f: \mathcal{D} \rightarrow \mathbb{S}^{n-1}$ on the space of databases $\mathcal{D}$. Note that in this case, we can use the sensitivity $\Delta$ of $f$ with respect to either $d_{\iota}$ (by Corollary 16) or $d_{2}$ (by Theorem 15).

3.2.1 VMF marginal distributions. By Lemma 8 and Corollary 9, the mixture and angular densities of a VMF distribution are

$$
\begin{aligned}
& \operatorname{VMFMix}[t]=C_{\mathrm{VMF}}^{\prime} \cdot\left(1-t^{2}\right)^{\frac{n-3}{2}} e^{\kappa t}, \\
& \operatorname{VMFArc}[\theta]=C_{\mathrm{VMF}}^{\prime} \cdot \sin ^{n-2}(\theta) e^{\kappa \cos (\theta),}
\end{aligned}
$$

where the normalization factor amounts to

$$
\begin{aligned}
C_{\mathrm{VMF}}^{\prime} & =C_{\mathrm{VMF}} \cdot S_{n-2} \\
& =\left(\frac{\kappa}{2}\right)^{v}\left(\Gamma\left(\frac{1}{2}\right) \Gamma\left(\frac{n-1}{2}\right) I_{v}(\kappa)\right)^{-1} \\
& =e^{\kappa} \cdot \mathrm{B}^{-1}\left(\frac{1}{2}, \frac{n-1}{2}\right) \cdot M^{-1}\left(\frac{n-1}{2} ; n-1 ; 2 \kappa\right) .
\end{aligned}
$$

The mixture density is used in the rejection sampling scheme for the VMF distribution by Ulrich [38] and Wood [44], and is based on earlier work by Saw [36]. We use it next for the expected distance.

3.2.2 Expected Euclidean distance. To assess the error induced by a mechanism, we can use statistical tools such as the expected value of an error measure based on the underlying distribution. Concretely, for a random vector $\mathbf{x} \sim \operatorname{VMF}(\boldsymbol{\mu}, \kappa)$, we provide an analytical expression for the expected L2 distance to the mode $\boldsymbol{\mu}$ :

Theorem 17. The expected Euclidean distance between a random vector $\mathbf{x} \sim \operatorname{VMF}(\boldsymbol{\mu}, \kappa)$ and the mode $\boldsymbol{\mu}$ can be expressed as expected value over the mixture density. It evaluates to

$$
\begin{aligned}
\underset{\mathbf{x} \sim \mathrm{VMF}}{\mathbb{E}}\left[d_{2}(\mathbf{x}, \boldsymbol{\mu})\right] & =\underset{t \sim \mathrm{VMFMix}}{\mathbb{E}}[\sqrt{2} \sqrt{1-t}] \\
& =\frac{\mathrm{B}\left(\frac{1}{2}, \frac{n}{2}\right)}{\mathrm{B}\left(\frac{1}{2}, n-\frac{1}{2}\right)} \frac{M\left(\frac{n-1}{2} ; n-\frac{1}{2} ; 2 \kappa\right)}{M\left(\frac{n-1}{2} ; n-1 ; 2 \kappa\right)} .
\end{aligned}
$$

3.2.3 Mixture CDF. Kurz and Hanebeck [27] provide analytical solutions for the CDF of the VMF angular distribution in the context of sampling. While their solution is an analytical, closed-form expression of elementary functions when $n$ is odd, it involves an infinite series in terms of special functions for even $n$.

In the following, we present a concise, analytic solution for the CDF of the VMF mixture distribution in terms of confluent hypergeometric series covering both odd and even dimensions:

Theorem 18. Setting $\alpha:=\frac{n-1}{2}$ and $x:=\frac{T+1}{2}$, the CDF of the VMF mixture distribution $\operatorname{VMFMix}(n, \kappa)$ at $T \in[-1,1]$ can be written as

$$
\operatorname{VMFMix}(n, \kappa)[t \leq T]=\frac{x^{\alpha}}{\alpha} \frac{\Phi_{1}(\alpha, 1-\alpha, 1+\alpha ; x, 2 \kappa x)}{\mathrm{B}(\alpha, \alpha) M(\alpha, 2 \alpha, 2 \kappa)} .
$$




\subsection{Purkayastha privacy mechanism}

The VMF distribution enjoys wide popularity among spherical distributions, and provides differential as well as $d_{2}$ - and $d_{<}$-privacy as shown in the previous section. However, we also observe potential shortcomings, namely the probability decreases exponentially with the squared L2 distance from the mode, i.e., the distance is measured as straight line through the sphere. Instead, we would rather have it decrease exponentially with the surface distance on the sphere, i.e., with $\arccos \left(\boldsymbol{\mu}^{\top} \boldsymbol{x}\right)$. It turns out that this is precisely the distribution in Definition 11 studied by Purkayastha [34]. We immediately obtain a corresponding Purkayastha privacy mechanism as follows:

Theorem 19 ( $\epsilon d_{L}$-privacy of Purkayastha mechanism). Let $\epsilon>0$ be a privacy parameter. The Purkayastha mechanism on $\mathbb{S}^{n-1}$ induced by $x \mapsto \operatorname{Pur}(x, \epsilon)$ for $x \in \mathbb{S}^{n-1}$ fulfills $\epsilon d_{\llcorner}$-privacy.

By Fact 14, the Purkayastha mechanism $\operatorname{Pur}(x, \epsilon / \Delta)$ also provides $\epsilon$-DP for sphere-valued functions $f: \mathcal{D} \rightarrow \mathbb{S}^{n-1}$ with $d_{\llcorner}$sensitivity $\Delta$ on the space of databases $\mathcal{D}$.

3.3.1 Purkayastha marginal densities. By Lemma 8 and Corollary 9 , we obtain the Purkayastha mixture and angular densities as

$$
\begin{aligned}
& \operatorname{PurMix}[t]=C_{\text {Pur }}^{\prime} \cdot\left(1-t^{2}\right)^{\frac{n-3}{2}} e^{-\kappa \arccos (t),} \\
& \operatorname{PurArc}[\theta]=C_{\text {Pur }}^{\prime} \cdot \sin ^{n-2}(\theta) e^{-\kappa \theta},
\end{aligned}
$$

with normalization factor $C_{\mathrm{Pur}}^{\prime}=C_{\mathrm{Pur}} \cdot S_{n-2}=F_{n-2,-\kappa}^{-1}(\pi)$.

Integrating the angular density. Having derived an expression for the angular density $\operatorname{PurArc}[\theta]$, we are interested in statistical properties such as its expected value to assess the average error, or the angular CDF PurArc $[\theta \leq \vartheta]$ which is fundamental for the sampling algorithm we propose in Section 3.4.2.

The angular density is specified through a function $e^{a x} \sin ^{n} x$, where $n \in \mathbb{N}$ and $a \in \mathbb{R}$. Gradshteyn and Ryzhik [15, 2.662] provide separate closed-form expressions for its antiderivative for even and odd $n$. We rewrite these expressions and provide the following unified solution which allows to efficiently evaluate such integrals:

Fact 20. An antiderivative of $e^{a x} \sin ^{n} x$ with $n \in \mathbb{N}$ and $a \in \mathbb{R}$ is

$$
E_{n, a}(x):=e^{a x} \sum_{k=0}^{m} \mathcal{C}_{k} \mathcal{T}_{k}(x),
$$

where $m=\lfloor n / 2\rfloor$,

$$
\begin{aligned}
C_{k} & =\frac{n !}{(n-2 k) !} \prod_{\ell=0}^{k} \frac{1}{\left(a^{2}+(n-2 \ell)^{2}\right)}, \quad \text { and } \\
\mathcal{T}_{k}(x) & =\sin ^{n-2 k-1}(x)[a \sin (x)-(n-2 k) \cos (x)] .
\end{aligned}
$$

In particular, the definite integral over $[0, r]$ is given by

$$
F_{n, a}(r):=\int_{0}^{r} e^{a x} \sin ^{n} x \mathrm{~d} x=E_{n, a}(r)-E_{n, a}(0) .
$$

A special case is the normalization factor $C_{\mathrm{Pur}}^{\prime}=F_{n-2,-\kappa}^{-1}(\pi)$ :

Lemma 21. The integral $F_{n, a}(\pi)=\int_{0}^{\pi} e^{a x} \sin ^{n} x \mathrm{~d} x$ evaluates to

$$
F_{n, a}(\pi)= \begin{cases}\frac{n !\left(e^{a \pi}-1\right)}{a\left(a^{2}+2^{2}\right)\left(a^{2}+4^{2}\right) \cdots\left(a^{2}+n^{2}\right)} & \text { for even } n, \\ \frac{n !\left(e^{a \pi}+1\right)}{\left(a^{2}+1^{2}\right)\left(a^{2}+3^{2}\right) \cdots\left(a^{2}+n^{2}\right)} & \text { for odd } n .\end{cases}
$$

3.3.2 Expected surface distance. We provide a closed-form solution for the expected angle of a Purkayastha random vector as follows:

Theorem 22. The expected surface distance (or angle) between a random point $\mathbf{x} \sim \operatorname{Pur}(\boldsymbol{\mu}, \kappa)$ and the mode $\boldsymbol{\mu} \in \mathbb{S}^{n-1}$ can be expressed as expected angular density. It evaluates to

$$
\begin{aligned}
\underset{\mathbf{x} \sim \operatorname{Pur}}{\mathbb{E}}\left[d_{L}(\mathbf{x}, \boldsymbol{\mu})\right] & =\underset{\theta \sim \operatorname{PurArc}}{\mathbb{E}}[\theta] \\
& =2 \kappa \sum_{\ell=1}^{m} A_{\ell}+ \begin{cases}\frac{\pi}{1-e^{\kappa \pi}}-\frac{1}{\kappa} & \text { ifn is even, } \\
\frac{\pi}{1+e^{\kappa \pi}} & \text { ifn is odd, }\end{cases}
\end{aligned}
$$

where $A_{\ell}:=\left(\kappa^{2}+(n-2 \ell)^{2}\right)^{-1}$ for $1 \leq \ell \leq m:=\left\lfloor\frac{n}{2}\right\rfloor$.

3.3.3 Angular CDF. We provide the following expression for the CDF of the angular distribution PurArc in terms of Eqs. (11) and (12):

Corollary 23. The CDF of the Purkayastha angular distribution $\operatorname{PurArc}(n, \kappa)$ is

$$
\operatorname{PurArc}[\theta \leq \vartheta]=C_{\text {Pur }}^{\prime} \int_{0}^{\vartheta} e^{-\kappa \theta} \sin ^{n-2}(\theta) \mathrm{d} \theta=\frac{F_{n-2,-\kappa}(\vartheta)}{F_{n-2,-\kappa}(\pi)}
$$

Note that this is a closed-form solution that can be efficiently evaluated in terms of finite sums $E_{n, a}(x)$ (Fact 20) and the formula for $F_{n, a}(\pi)$ (Lemma 21) for both odd and even $n$. This is crucial for the Purkayastha sampling method we develop in the next section.

\subsection{Sampling algorithms}

In this section, we discuss concrete algorithms for our directional privacy mechanisms, i.e., to generate samples from the underlying distributions. For some general intuition on sampling rotationally symmetric distributions, we refer the reader to Section 2.2.1. Due to its popularity, the VMF distribution has been studied extensively, and proven sampling methods already have been published; two of them we describe in Section 3.4.1. In contrast, no methods have been published so far for the Purkayastha distribution. Therefore, in Section 3.4.2, we contribute the first sampling algorithm for the Purkayastha distribution.

3.4.1 VMF sampling methods. To generate a point $\mathrm{x} \sim \operatorname{VMF}(\boldsymbol{\mu}, \kappa)$, we can employ the existing rejection scheme by Ulrich [38] and Wood [44]: Pursuant to Section 2.2.1, it involves two crucial steps: First, the tangent-normal decomposition $\mathbf{x}=t \boldsymbol{\mu}+\sqrt{1-t^{2}} \boldsymbol{\xi}$ in Eq. (1) reduces the multivariate sampling problem to a univariate one, namely sampling $t \backsim \operatorname{VMFMix}(n, \kappa)$ from the mixture distribution, as well as a direction vector $\xi \backsim U n i\left(\mathbb{S}^{n-2} \perp \boldsymbol{\mu}\right)$. This avoids the curse of dimensionality since the mixture density is onedimensional, and uniform samples from a hypersphere are easily created by normalizing samples from a (multivariate) standard normal distribution. Second, we need an efficient sampling algorithm for the reduced problem. A clever way to solve this is the rejection method [44, Algorithm $\left.\mathrm{VM}^{*}\right]$ for $\operatorname{VMFMix}(n, \kappa)$. Ulrich [38] showed that the acceptance ratio is at least $\approx 66 \%$ for any parameters $n$ and $\kappa$, resulting in a very efficient method even in high dimensions.

More recently, Kurz and Hanebeck [27] proposed another sampling algorithm for the VMF distribution that is best described as approximate inversion method. It works by substituting $t=\cos (\vartheta)$ in the tangent-normal decomposition as in Eq. (2) and constructing 


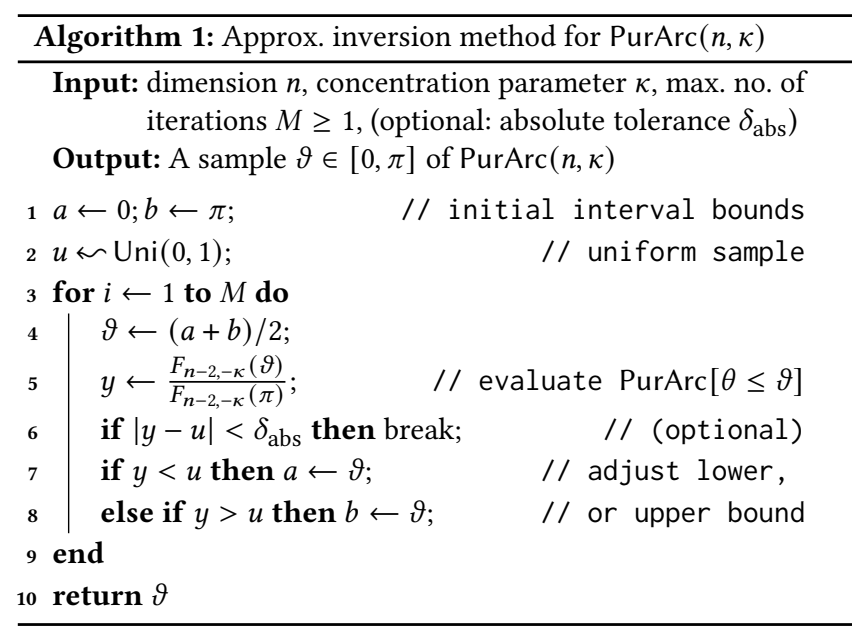

a sample $\mathbf{x}=\cos (\vartheta) \boldsymbol{\mu}+\sin (\vartheta) \xi$. This reduces the problem to generating $\vartheta \backsim \operatorname{VMFArc}(n, \kappa)$ from the univariate angular distribution. If the corresponding angular $\operatorname{CDF} \operatorname{VMFArc}(n, \kappa)[\theta \leq \vartheta]$ was invertible analytically, a textbook version of the inversion method (see, e.g., [8]) could be used to sample $\vartheta$. Kurz and Hanebeck solve this by approximately inverting the CDF: If we can efficiently compute the $\operatorname{CDF} u=\operatorname{VMFArc}(n, \kappa)[\theta \leq \vartheta]$, we can approximate its inverse $\vartheta=\operatorname{VMFArc}(n, \kappa)^{-1}[u]$ numerically, e.g., by interval bisection, which is "guaranteed to converge up to machine precision" in a reasonable number of steps [27]. Unfortunately, their solution for $\operatorname{VMFArc}(n, \kappa)[\theta \leq \vartheta]$ is analytical only for odd $n$, while it contains an infinite series in terms of special functions for even $n$ which we cannot evaluate efficiently. Therefore, this approach is only viable for VMF when $n$ is odd, which is why we prefer the rejection scheme from the previous paragraph as it is fast and simple to use in general. However, we show next that this idea is useful for sampling the Purkayastha distribution.

3.4.2 Purkayastha sampling method. To our best knowledge, there is no published sampling method for the Purkayastha distribution. Cutting et al. [7] state that they generated samples for lower dimensions up to $n=100$, but without specifying the exact method they used. Rather, they give the following explanation (emphasis ours):

The Purkayastha distribution is numerically hard to generate for dimensions larger than 150, which is the only reason why the dimensions considered in this second simulation are smaller than in the first one.

Here, the "first" and "second simulation" refer to sampling from the VMF and Purkayastha distribution, respectively.

Approximate inversion Purkayastha sampling algorithm. Recall that we have derived a solution for the angular CDF of the Purkayastha distribution, $\operatorname{PurArc}(n, \kappa)[\theta \leq \vartheta]=F_{n-2,-\kappa}(\vartheta) / F_{n-2,-\kappa}(\pi)$, in Corollary 23. While we are not aware of a way to directly compute its inverse to apply the inversion method [8] for $n>2$, the solution itself is a finite closed-form expression that can be computed analytically. We hence propose an approximate inversion method, similar to the approach by Kurz and Hanebeck [27] for VMF in Section 3.4.1, to obtain a new Purkayastha sampling algorithm: Since we can efficiently compute the angular $\operatorname{CDF} u=\operatorname{PurArc}(n, \kappa)[\theta \leq \vartheta]$, we can approximate its inverse $\vartheta=\operatorname{PurArc}(n, \kappa)^{-1}[u]$ numerically. We describe the core method to sample $\vartheta \backsim \operatorname{PurArc}(n, \kappa)$ in Algorithm 1. Once we have a sample $\vartheta$, we draw $\xi \sim \operatorname{Uni}\left(\mathbb{S}^{n-2} \perp \boldsymbol{\mu}\right)$ and as above use the tangent-normal decomposition Eq. (2) to construct

$$
\mathbf{x}=\cos (\vartheta) \boldsymbol{\mu}+\sin (\vartheta) \xi \sim \operatorname{Pur}(\boldsymbol{\mu}, \kappa)
$$

Since our solution for the angular CDF is a closed-form expression with finitely many terms in any number of dimensions $n$, we argue that our approximate inversion method for the Purkayastha distribution is practical regardless of the parity of $n$.

Algorithm 1 can easily be vectorized to generate multiple samples at once, or parallelized to utilize multiple CPU cores. In fact, we benchmark our method in up to tens of thousands of dimensions (cf. Section 4.1), pushing beyond the status quo [7] by providing an efficient sampling algorithm in dimensions much larger than 150.

\subsection{Choice of parameters based on privacy level}

To actually run the proposed directional privacy mechanisms on a given input vector $x \in \mathbb{S}^{n-1}$, we need to generate samples from $\operatorname{Pur}(x, \kappa)$ or $\operatorname{VMF}(x, \kappa)$ where the mode is given by the input $x$ and the concentration parameter $\kappa$ is defined through the privacy parameter $\epsilon$. Having described sampling methods for both the VMF mechanism (cf. Section 3.4.1) and a novel sampling scheme for the Purkayastha mechanism (cf. Section 3.4.2), it remains to explain the exact choice of $\kappa$ based on $\epsilon$ and the desired notion of privacy.

Given a unit vector $x \in \mathbb{S}^{n-1}$, in order to achieve directional privacy with privacy parameter $\epsilon$, i.e. $\epsilon d_{L}$-privacy (Definition 13), we simply need to set $\kappa=\epsilon$ and draw a sample $\mathrm{z} \backsim \operatorname{Pur}(x, \epsilon)$ or $\mathrm{z} \backsim \operatorname{VMF}(x, \epsilon)$ as shown in Corollary 16 and 19 , respectively.

Metric privacy (Definition 3) [4] and its variants can also be interpreted as providing a privacy (or indistinguishability) level $\ell=\epsilon r$ to any two points $x, x^{\prime}$ within a protection radius (or angle) $r>0$, cf. [2]. In case of directional privacy (Definition 13), this is achieved by sampling with $\kappa=\ell / r$. In other words, an $(\ell / r)$-private mechanism achieves a privacy level $\ell$ within a protection radius $r$.

As special case, when $x=f(D)$ is the result of a (query) function $f: \mathcal{D} \rightarrow \mathbb{S}^{n-1}$, we achieve pure $\epsilon$-DP by setting the protection radius $r:=\Delta$ to the (worst-case) sensitivity of $f$, i.e., by sampling with a concentration parameter $\kappa=\epsilon / \Delta$ as per Fact 14 . Thus, directional privacy allows relaxing pure DP by specifying a protection radius $r$ smaller than the sensitivity $\Delta$.

\subsection{Circular and spherical baselines}

For comparison, we consider the following adaptions of established standard privacy mechanisms to directional data. The first and second mechanisms, Clipped and Wrapped Laplace, are suitable for circular data $(n=2)$, whereas the third one, Polar Laplace, can be regarded as variant of Wrapped Laplace for spherical data $(n=3)$.

3.6.1 Clipped Laplace. A straightforward application of the usual Laplace mechanism [10] with post-processing achieves DP on the circle by adding Laplace noise to a given angle, followed by clipping the result to an interval covering one full circle, say $[0,2 \pi)$ or $[-\pi, \pi)$. This method is simple, but clearly has drawbacks: For small $\epsilon$, the major part of the probability mass will be outside the 


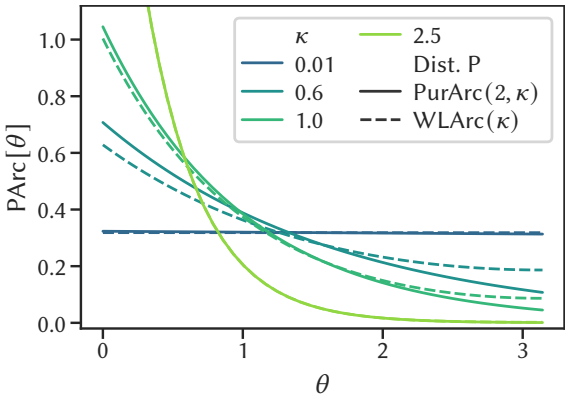

(a) Angular PDFs on $\mathbb{S}^{1}$ (circular case, $n=2$ )

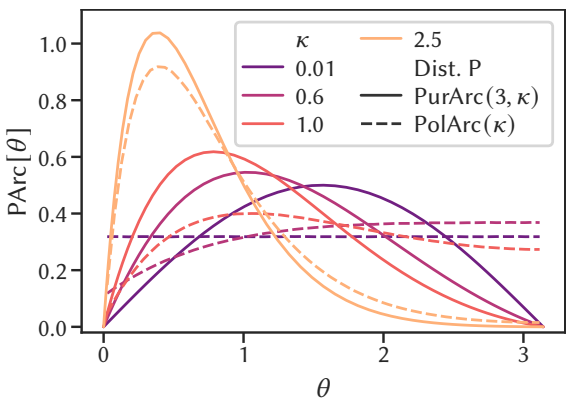

(b) Angular PDFs on $\mathbb{S}^{2}$ (spherical case, $n=3$ )

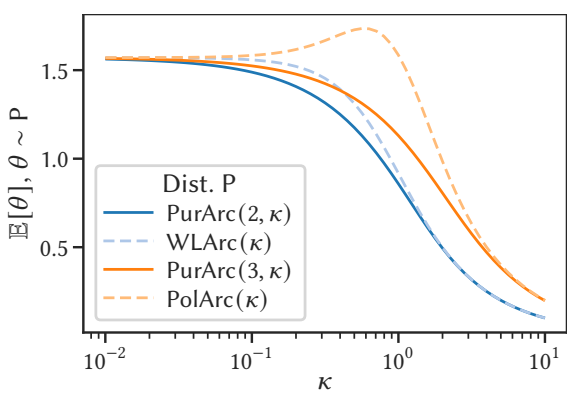

(c) Expected angles: larger for baselines

Figure 2: Comparison of angular densities and expectations of Purkayastha vs. Wrapped/Polar Laplace (solid vs. dashed lines).

clipping range, creating a bias towards the angle at its boundaries. We therefore use it only in selected experiments.

3.6.2 Wrapped Laplace. Instead of clipping, we can add Laplace noise to the original angle $\alpha$ and wrap it around the circle by reducing the result modulo $2 \pi$. This results in a so-called (symmetric) Wrapped Laplace (WL) distribution with mean $\alpha$. With the usual parametrization on the unit circle, the density of a WL distribution with zero mean and concentration parameter $\kappa \geq 0$ is (cf. [21])

$$
\mathrm{WL}(\kappa)[\theta]=\frac{\kappa}{2}\left(\frac{e^{-\kappa \theta}}{1-e^{-\kappa 2 \pi}}+\frac{e^{\kappa \theta}}{e^{\kappa 2 \pi}-1}\right), \quad \theta \in[0,2 \pi) .
$$

Angular density. In accordance with Corollary 9, the corresponding angular density WLArc is the density of points with the same angle from the mean, in any direction. That is, it identifies an angle $\theta \in[0, \pi)$ with its mirror image $2 \pi-\theta$. By symmetry, it is just twice the density on the full circle:

$$
\operatorname{WLArc}(\kappa)[\theta]=\kappa\left(\frac{e^{-\kappa \theta}}{1-e^{-2 \kappa \pi}}+\frac{e^{\kappa \theta}}{e^{2 \kappa \pi}-1}\right), \quad \theta \in[0, \pi) .
$$

While the Purkayastha angular density $\operatorname{PurArc}(2, \kappa)[\theta] \propto e^{-\kappa \theta}$ on $\mathbb{S}^{1}$ only has a single term $e^{-\kappa \theta}$, cf. Eq. (9), $\operatorname{WLArc}(\kappa)$ has an additional second term $e^{+\kappa \theta}$ that increases with the angle $\theta$. The wrapping hence smoothens the distribution by moving probability mass away from the mode as illustrated in Fig. 2a. It hence provides less accuracy than Purkayastha at the same privacy level, thus motivating the need for specialized directional mechanisms.

Expected angular distance. Similarly to the derivation of the expected surface distance for the Purkayastha distribution from PurArc $[\theta]$, we can derive the expected angular distance for the WL distribution from WLArc $[\theta]$. The result is

$$
\underset{\theta \sim \operatorname{WLArc}(\kappa)}{\mathbb{E}}[\theta]=\frac{1}{\kappa}\left(\frac{1}{1+e^{-\kappa \pi}}-\frac{1}{1+e^{\kappa \pi}}\right)=\frac{1}{\kappa} \frac{1-e^{-\kappa \pi}}{1+e^{-\kappa \pi}} .
$$

For comparison, the expected angular distance of the circular Purkayastha distribution from Lemma 21 simplifies to

$$
\underset{\theta \sim \operatorname{PurArc}(2, \kappa)}{\mathbb{E}}[\theta]=\frac{1}{\kappa}-\frac{\pi}{e^{\kappa \pi}-1}=\frac{1}{\kappa}-\frac{\pi e^{-\kappa \pi}}{1-e^{-\kappa \pi}} .
$$

The formula for the expected angular distances allow us to analytically compare the average (angular) error induced by the distributions based on the concentration parameter $\kappa$, which in turn depends on the privacy parameter $\epsilon$ (cf. Section 3.5):
Theorem 24. For any value $\kappa>0$, the WL distribution has a strictly larger expected angular distance than Purkayastha:

$$
\underset{\theta \sim \text { WLArc }}{\mathbb{E}}[\theta]>\underset{\theta \sim \operatorname{PurArc}}{\mathbb{E}}[\theta]+\frac{\kappa \pi^{2}}{e^{2 \kappa \pi}-1}>\underset{\theta \sim \operatorname{PurArc}}{\mathbb{E}}[\theta]
$$

The limits of both expected distances are 0 as $\kappa \rightarrow \infty$ and $\frac{\pi}{2}$ as $\kappa \rightarrow 0$.

Figure 2c shows expected angles of $\operatorname{PurArc}(2, \kappa)$ and $\operatorname{WLArc}(\kappa)$ (blue lines) for a range of $\kappa \in\left[10^{-2}, 10\right]$. As we can see, the baseline has larger expected errors which is in line with Theorem 24.

3.6.3 Polar Laplace. The Planar Laplace (PL) mechanism [2, 4] was originally invented in the context of protecting geolocation data. It can be considered as a two-dimensional variant of the standard Laplace mechanism that works in Cartesian coordinates by translating the initial starting point $x \in \mathbb{R}^{2}$ by a certain distance $r$ along a certain direction $\alpha$. The distance $r$ and direction $\alpha$ are polar coordinates obtained by sampling a random direction $\alpha \sim \operatorname{Uni}(0,2 \pi)$ and a displacement radius $r \sim \Gamma(2,1 / \epsilon)$ from a Gamma distribution.

When applying the PL mechanism to spherical instead of Cartesian coordinates, we obtain the Polar Laplace mechanism ${ }^{1}$ [5] that respects the curvature of the (roughly) spherical Earth: The initial point $x$ is represented in spherical coordinates (e.g., latitude and longitude). We then draw a random sample of polar coordinates $(r, \alpha) \sim \Gamma(2,1 / \epsilon) \times \operatorname{Uni}(0,2 \pi)$ as with PL, and, as post-processing step, solve the direct geodesic problem ${ }^{2}$ to find the destination point $\mathrm{z}$ that is reached after traveling for a distance of $r$ units in the direction specified by $\alpha$. As with WL, we pass the starting point again every time a distance equal to the circumference of the sphere has been traversed; therefore, Polar Laplace can be regarded as two-dimensional variant of the WL mechanism (cf. Section 3.6.2).

Angular density and expected distance. In order to compare the Polar Laplace and Purkayastha mechanisms on the sphere $\mathbb{S}^{2}$, we again use their angular densities as auxiliary. We simulated 64M samples to approximate the angular density $\operatorname{Pol} \operatorname{Arc}(\kappa)[\theta]$ and its expected value for $\theta$. We compare it with the (exact) solutions for the three-dimensional Purkayastha angular density $\operatorname{PurArc}(3, \kappa)[\theta]$ and its expected value, as provided in Eq. (9) and Theorem 22.

Figure $2 \mathrm{~b}$ shows the angular densities of the Purkayastha and Polar distributions. For all values of $\kappa, \operatorname{PurArc}(3, \kappa)[\theta]$ is higher near $\theta=0$ and approaches 0 as $\theta \rightarrow \pi$, whereas $\operatorname{Pol} \operatorname{Arc}(\kappa)[\pi]$ is

\footnotetext{
${ }^{1}$ Implementation in laplace. js at https://github.com/chatziko/location-guard. ${ }^{2}$ Solution formula from https://www.movable-type.co.uk/scripts/latlong.html.
} 


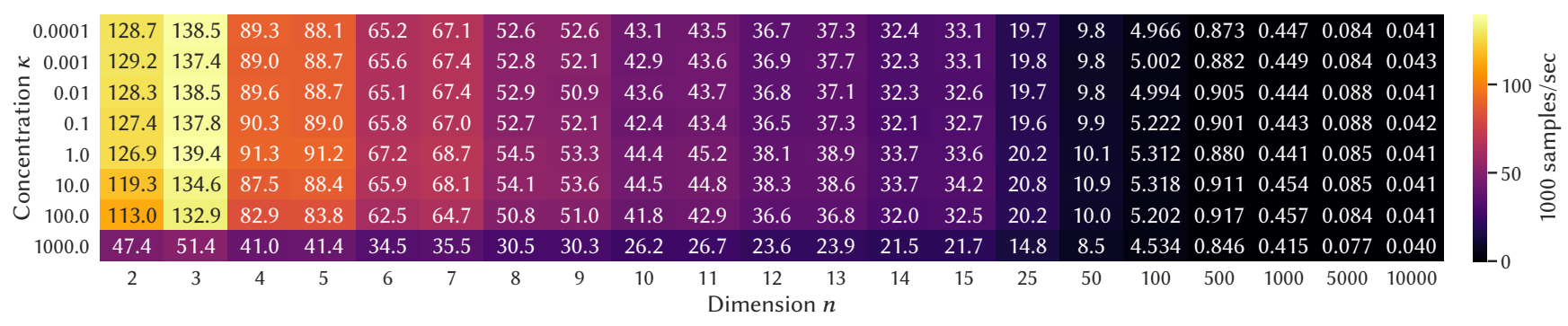

Figure 3: Sampling rate $\left(\times 10^{3}\right)$ of the Purkayastha approximate inversion method (Algorithm 1) with various parameters.

strictly above 0 . The expected angles of both spherical distributions are shown in Figure 2c (orange lines) and approach 0 for $\kappa \rightarrow \pi$. As $\kappa$ decreases from $\pi$ to $0, \mathbb{E}_{\theta \sim \operatorname{PurArc}}[\theta]$ steadily rises to $\frac{\pi}{2}$ and approaches the uniform distribution. In contrast, $\mathbb{E}_{\theta \sim \operatorname{Pol} A r c}[\theta]$ goes up to over 1.7 at $\kappa \approx 0.6$ (i.e., worse than the uniform distribution), and only then falls back to $\frac{\pi}{2}$, which is quite remarkable.

To explain this phenomenon, consider the expected displacement radius which amounts to $\mathbb{E}_{r \sim \Gamma(2,1 / \kappa)}[r]=\frac{2}{\kappa}$. For $\kappa \approx \frac{2}{\pi} \approx 0.637$, it is close to $\pi$, which is the farthest distance we can go from $x$ to its antipodal point $-x$ on $\mathbb{S}^{2}$; consequently, most random points will end up on the "wrong" hemisphere. This raises the expected angle $\mathbb{E}_{\theta \sim \operatorname{PolArc}}[\theta] \approx 1.733$ to over $\frac{\pi}{2}$ for such $\kappa$, indicating a point of no return where the distribution's mode reverses from $x$ to $-x$. Overall, these results indicate an advantage for Purkayastha over Polar Laplace, particularly for $\kappa \approx \frac{2}{\pi}$.

\section{EXPERIMENTS}

In this section, we experimentally verify the proposed methods. We start by testing the efficiency of our novel Purkayastha sampling algorithm, which is crucial for the Purkayastha mechanism. We then apply our methods to real-world data: First, we analyze the impact of the privacy mechanisms on the circular mean and ranking statistics. Next, we consider temporal and spatial histograms from periodic times-of-day and geolocations on a spherical coordinate system. Finally, we compute "busyness" histograms indicating the activity or popularity of certain locations, such as stores or restaurants, over the course of a day, through a combined application of directional privacy mechanisms to both spatial and temporal check-in data.

Implementation. We use Python 3 for our experiments. Arithmetic and computations are based on numpy $[32,41]$ and scipy [39]. For confluent hypergeometric and special functions, we rely on the mpmath multi-precision library [22]. We implemented both sampling algorithms, Algorithm 1 for Purkayastha and the VMF rejection method by Ulrich [38] and Wood [44], with basic optimizations such as vectorization and JIT compilation via Numba [28].

\subsection{Sampling efficiency}

To measure the efficiency of our proposed Purkayastha approximate inversion method, we run our implementation of Algorithm 1 with varying $n$ and $\kappa$ for at least 60 seconds and count the number of generated samples. Based on the counts and elapsed times, we compute the individual rate of samples per second. While single-threaded, our implementation uses vectorization to work on multiple samples simultaneously. The experiments were run in parallel on a 48-core Xeon Platinum 8259CL system with each instance corresponding to one parametrization $(n, \kappa)$ of the PurArc distribution.

Results. Figure 3 shows the achieved sampling rate of our Purkayastha approximate inversion method in thousands of samples per second. We push beyond the status quo [7] by generating samples even in thousands of dimensions. Clearly, the rate decreases with the dimensionality $n$ due to the increasing number of terms in Eq. (10) that is used to compute $\operatorname{PurArc}(n, \kappa)[\theta \leq \vartheta]$ (Corollary 23). Another factor is the concentration parameter $\kappa$ : Larger values decrease the sampling rate first slightly, and then more pronounced for $\kappa \gtrsim 100$. However, with DP, we typically prefer low privacy losses $\epsilon$ that correspond to small values of $\kappa$ (cf. Section 3.5)-and thus yield higher speeds.

Sampling rates of tens to over hundreds of thousands samples per second clearly show that the Purkayastha approximate inversion method is practical in the low- to medium-dimensional setting. As the dimensionality $n$ gets larger, however, the sampling rate decreases steadily until it will eventually become too low for the method to be practical. As this is an intrinsic issue with the method being based on formula whose complexity increases with $n$, it leaves room for further research. Still, practical improvements to the current approach are possible, for instance by porting the Python code to a native language like $\mathrm{C}$ or parallelization on multiple cores. Lastly, we note that even fewer than hundreds of samples per second may be sufficient for many real-world applications, particularly in the local model where each participant perturbs just their own data (i.e., only few samples) prior to submitting it to a central server.

\subsection{Circular mean on periodic data}

The National Sleep Foundation (NSF) regularly conducts surveys of US citizens on their sleep habits including questions on their bed and wake times. Among the key reported figures in the surveys' findings are the average wake and bed times; these times-of-day are periodic on a 24-hour scale and hence provide a natural example of directional data that is suitable for directional privacy.

Scenario and privacy models. Suppose we work for a polling agency that wants to conduct a similar survey of sleeping habits, but with formal privacy guarantees as offered by differential privacy. The survey results with statistics such as average bed and wake times shall be made public or shared with another third party. We can distinguish two major approaches corresponding to the central and local privacy models introduced in Section 2.1. 
In the central model, the survey participants trust the polling agency to handle their sensitive data confidentially. Hence, they faithfully report their unaltered answers to the agency. After collection of all survey responses, the agency prepares the statistics from the original data and applies appropriate privacy mechanisms to sanitize the results, which can then be shared or made public.

The local model can provide a suitable alternative if the survey participants do not trust the polling agency: Instead of providing faithful answers, the respondents first sanitize their answers themselves before reporting the altered responses back to the agency. From the collected obfuscated responses, the agency computes the desired statistics that can be publicized afterwards.

Circular statistics. When taking the average or difference of periodic data, it is not sufficient to simply take the arithmetic mean or absolute distances. Instead, we must use periodic variants such as the circular mean which works by averaging the direction vectors, or the circular distance which takes the shortest path in any direction, clock- or counterclockwise, so two times differ by at most 12 hours.

Let $\boldsymbol{t}=\left(t_{1} \ldots, t_{N}\right)$ be a sequence of real numbers. We write the usual arithmetic mean of $\boldsymbol{t}$ as $\varnothing(\boldsymbol{t})=\varnothing\left(t_{1}, \ldots, t_{N}\right):=\frac{1}{N} \sum_{i} t_{i}$. Now let us assume $t$ is periodic with period (circumference of the circle) $p>0$, i.e., each $t_{i} \in[0, p)$. Then the circular mean of $t$ is

$$
\varnothing_{p}(\boldsymbol{t})=\varnothing_{p}\left(t_{1}, \ldots, t_{N}\right):=\frac{p}{2 \pi} \arctan 2\left(\varnothing\left(\sin \frac{2 \pi \boldsymbol{t}}{p}\right), \varnothing\left(\cos \frac{2 \pi \boldsymbol{t}}{p}\right)\right) .
$$

The circular difference between $p$-periodic values $s$ and $t$ is

$$
\delta_{p}(s, t):= \begin{cases}p-(s-t) & \text { if } s-t>p / 2, \\ p+(s-t) & \text { if } s-t<-p / 2, \\ s-t & \text { otherwise, }\end{cases}
$$

i.e., the signed difference equal to the positive clock- or negative counterclockwise arc length from $t$ to $s$ taking values in $[-p, p]$. The circular distance between $s$ and $t$ with values in $[0, p]$ is

$$
d_{p}(s, t):=\left|\delta_{p}(s, t)\right| .
$$

Note that the circular mean is highly sensitive to a change in the input: We can always construct a sequence $t_{1} \ldots, t_{N}$ so that changing a single $t_{i}$ will also cause the mean to point into the opposite direction. For instance, given a circle with period $2 \pi$, let $\left(t_{1}, t_{2}, t_{3}\right)=\left(\alpha, \pi-\alpha, \pm \frac{\pi}{2}\right)$ for some small $\alpha>0$. Therefore, we obtain the same sensitivity $\Delta_{\measuredangle}=\pi$ in the central and local model.

4.2.1 Local model advantage and sample complexity. Note that the central model normally has a lower sensitivity, so it injects less noise and hence is more accurate than the local model. However, in case of the circular mean, we anticipate an advantage for the local model: Given a sufficient amount of noisy responses, the locally injected noise will gradually cancel out, resulting in more accurate statistics than in the central model. This is similar to the mean of i.i.d. Gaussians which has a lower variance than each Gaussian on its own. Moreover, the local model can be used in scenarios where the data curator cannot be or is not trusted by the participants.

To examine this effect in case of the circular (and spherical) mean, we perform the following simulation experiment to determine the number of samples required to reach a certain accuracy: Let $t_{i, j} \sim \mathrm{P}$ denote i.i.d. samples from a circular distribution $\mathrm{P}$ with mean $\mu=0$
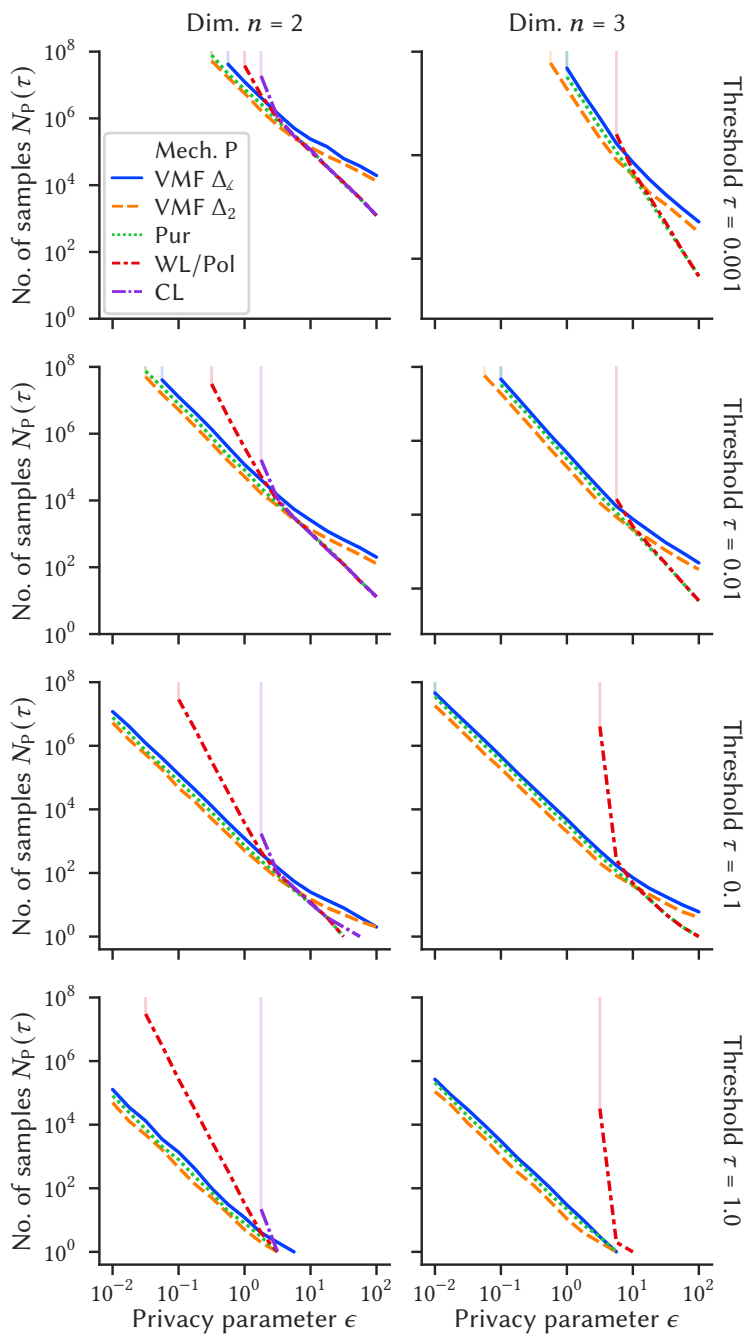

Figure 4: Sample complexity (mean over $R=1000$ runs).

$(1 \leq i \leq N, 1 \leq j \leq R)$. The number of samples required to maintain an average error below a given threshold $\tau \in[0, \pi]$ is

$$
N_{\mathrm{P}}(\tau):=\min \left\{k \in \mathbb{N}: \frac{1}{R} \sum_{j=1}^{R} \varnothing_{2 \pi}\left(t_{1, j}, \ldots, t_{i, j}\right) \leq \tau \forall i \geq k\right\},
$$

which we call the average sample complexity of $\mathrm{P}$ at $\tau$ over $R$ runs.

Results. Figure 4 shows the sample complexity for the circular ( $n=2$, left) and spherical ( $n=3$, right) VMF, Purkayastha, as well as the Clipped and Wrapped $(n=2)$ or Polar $(n=3)$ Laplace mechanisms with sensitivity $\Delta=\pi$ over $R=1000$ runs with $N=$ $10^{8}$ samples each. For large $\epsilon$, Purkayastha and the Laplace baselines approximately require the same number of samples to reach a given threshold $\tau$. For small $\epsilon$, VMF has a slight advantage over Purkayastha since it can be used with the smaller $d_{2}$-sensitivity under pure DP. In this case, all directional mechanisms show a similar, gentle slope and clearly outperform the Laplace baselines, where CL performs worst. In fact, even $10^{8}$ samples quickly become insufficient to reach the given thresholds for Polar Laplace in $n=3$ dimensions (thin vertical lines). This relates to Section 3.6.3, where 
we observe that Polar Laplace reaches an expected angle $1.733>\frac{\pi}{2}$ for $\kappa \approx \frac{2}{\pi}$ (i.e. $\epsilon=\kappa \Delta_{\measuredangle} \approx 2$ ). The behavior of wrapped distributions thus causes utility worse than a uniform distribution.

For polls like the NSF survey, the impact is significant: To reach an error below 0.1 with $\epsilon=1.0$, the service provider only needs to collect about 750 responses with Purkayastha instead of over 3600 with Wrapped Laplace, which represents an over 4.8-fold reduction. Conversely, given the same number of responses, our mechanisms achieve lower errors and higher accuracy as we see in the next Section 4.2.2. This makes the use of local DP practical under stricter conditions even with small privacy parameters $\epsilon \leq 10$.

4.2.2 Sleep study: average wake times and ranking statistics. In the following experiments, we simulate a privacy-preserving survey in a real-world setting, in both the central and local privacy model.

Dataset description. We rely on the NSF's 2011 dataset [14], which includes a total of 1,508 survey responses. The questions include their bed and wake times, both on workdays and weekends. The respondents are divided into four age groups: Baby Boomers (46-64), Generation X (30-45), Y (19-29), and Z (13-18 years).

Sanitization procedure and parameters. To sanitize the times-ofday reported in the survey on a 24-hour scale, we need to express them as 2-dimensional unit vectors that we can use as mode of the VMF or Purkayastha distribution. This is easily achieved by assigning to each hour the corresponding angle (in radians) on a 24-hour clock, and then transforming these angles to coordinates via sine and cosine. Conversely, after perturbing the points with one of our new mechanisms, we transform the points back to the 24-hour scale using the inverse trigonometric arctangent function.

For comparison, we also perturb the scalar data directly on the 24hour scale by means of the standard Laplace mechanism [10]. Since Laplace noise can be arbitrarily positive or negative, we reduce the perturbed values modulo 24 to map the values back into the domain $[0,24)$. In fact, this corresponds to a WL distribution on a 24-hour scale, as discussed in Section 3.6.1.

Let $\boldsymbol{t}=\left(t_{1}, \ldots, t_{N}\right)$ be the true times-of-day from the $N$ participants. Depending on the privacy model, we proceed as follows: In the central model, we take the average $\bar{t}=\varnothing_{24}(\boldsymbol{t})$ of all truly reported times, and then perturb $\bar{t}$ using one of the privacy mechanisms. In the local model, we first perturb each participant's value $t_{i}$ individually. Then, we compute the average from the perturbed values. The $d_{\measuredangle}$-sensitivity of the circular mean is $\Delta_{\measuredangle}=\pi$ radians, corresponding to 12 hours, even in the central model as changing a single input can cause the mean to flip to the opposite direction in the worst case. For VMF, we can also use the smaller $d_{2}$-sensitivity $\Delta_{2}=2$ corresponding to the diameter of the unit circle.

For each mechanism, VMF, Purkayastha, and Laplace, we vary the privacy parameter $\epsilon \in\left\{10^{k} \mid-4 \leq k \leq 3\right\}$ with step size $\Delta k=$ 0.2 . To stabilize the results, we repeat this procedure in each setting (privacy model, mechanism, and parameters) for $R=10000$ runs, so we obtain a sequence $\tilde{\boldsymbol{t}}=\left(\tilde{t}_{1}, \ldots, \tilde{t}_{R}\right)$ of anonymized average times.

Error evaluation for circular mean. We take each anonymized time $\tilde{t}_{i}$, whose mean we denote by $\tilde{t}=\varnothing_{24}(\tilde{\boldsymbol{t}})$, as estimate for the true average time $\bar{t}$. We want to estimate the error induced by the various privacy mechanisms on the average wake time compared

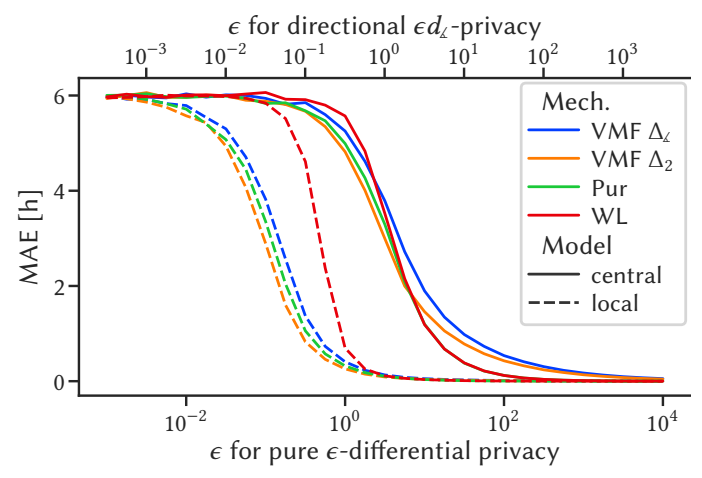

(a) MAE over various values of $\epsilon$ under directional and pure differential privacy (indicated by the top and bottom axis, respectively).

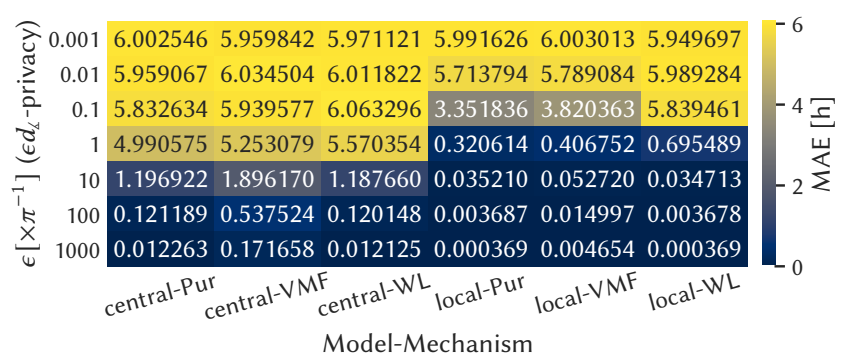

(b) Exemplary MAE values for various settings of the mechanisms (directional privacy; central and local model in cols. 1-3 and 4-6).

Figure 5: Comparison of the mean absolute error (MAE) between original and perturbed average wake times.

to the original, unperturbed data. To this end, we chose the mean absolute error (MAE), which is normally defined as $\frac{1}{R} \sum_{i=1}^{N}\left|\tilde{t}_{i}-\bar{t}\right|$. However, as noted earlier, we work with periodic data, so we must adapt the usual expression to its circular variant $\varnothing\left(d_{p}(\tilde{\boldsymbol{t}}, \bar{t})\right)$.

Figure $5 \mathrm{a}$ shows the MAE of the average wake time based on the original and perturbed values. In the local model (dashed lines), both directional privacy mechanisms clearly outperform WL across the entire range of privacy parameters $\epsilon$. For directional privacy (top scale), Purkayastha shows the lowest errors due to its higher concentration at the mode. However, for pure DP (bottom scale), VMF can be employed with smaller $d_{2}$-sensitivity $\Delta_{2}=2<\pi=\Delta_{\measuredangle}$ (orange line), which even outperforms Purkayastha in that case. In the central model (solid lines), WL and Purkayastha perform similarly well for large $\epsilon$ where VMF performs worst. However, in the strong privacy domain with small $\epsilon$, WL is worst, with Purkayastha providing the best directional privacy guarantees and VMF with the reduced $d_{2}$-sensitivity yielding the best differential privacy guarantees for $\epsilon \lesssim 10^{0.25}$. Figure 5b lists exemplary MAE values specifically for directional privacy to support these observations with concrete numbers.

Strikingly, the local model outperforms the central one in this experiment, which confirms what we anticipated in Section 4.2.1: The sensitivity of the circular mean is the same in both privacy models, where the locally injected noise gradually cancels out when many responses are averaged together, yielding lower errors. In both models, Purkayastha and VMF reach the lowest errors for a given directional and differential privacy parameter $\epsilon$, respectively. 


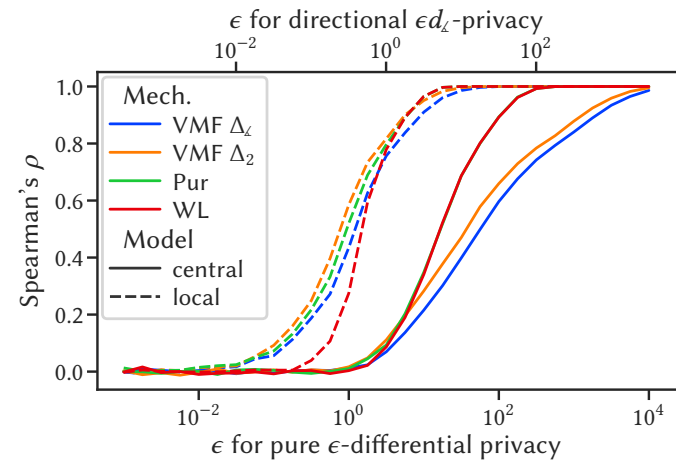

Figure 6: Comparison of Spearman's $\rho$ across the four age groups, over $\epsilon$ under directional and pure differential privacy (indicated by the top and bottom axis, respectively).

Ranking statistics. In the context of the NSF's sleep study, one aspect is to compare the wake (or bed) times among different groups, and determine, e.g., who gets up first or goes to bed latest. Concretely, let us suppose we want to infer the order of wakeup times among the four age groups (Generation-X, -Y, $-Z$, and Baby Boomers) from the survey data. As non-private baseline, we compute the average wake-up time for each group on the original dataset, and from there determine the ranking of the groups. We then simulate the survey being conducted in both the central and local privacy models as before, and determine the ranking of the age groups from the sanitized average wake-up times. To measure the impact of the privacy mechanisms on such statistics, we compute Spearman's rank correlation coefficient (also called Spearman's $\rho$ ) between the perturbed and original ranking of the four age groups.

Figure 6 shows Spearman's rank correlation coefficient $\rho$ (averaged over all runs) for the different mechanisms over the parameter range of $\epsilon$ and both privacy models. As we can see, the observations on the rank correlation are in line with the observations on the mean absolute errors reported in the previous experiment.

In the central model, Purkayastha and Wrapped Laplace (WL) (overlapping green and red lines) achieve similar $\rho$ values and both outperform VMF at virtually any given privacy level $\epsilon$ under both directional and differential privacy. However, in a small range of $\epsilon$ just below 1, Purkayastha shows higher correlation than WL, and VMF with the $d_{2}$-sensitivity also overtakes WL under pure DP.

The local model generally shows a better privacy-utility trade-off than in the previous results. Notably, Purkayastha appears to reach the highest correlation values among the three mechanisms under directional privacy, at virtually any given privacy level, which is well observable for $10^{-3} \lesssim \epsilon \lesssim 1$. Under pure DP, the VMF mechanism with the $d_{2}$-sensitivity stands out again and achieves even higher correlation scores than Purkayastha.

\subsection{Private histograms for spatio-temporal data}

Histograms and heatmaps are practical tools to visualize and interpret empirical data, particularly in one or two dimensions.
Scenarios. Suppose a location-based service, such as Google Maps or Foursquare, wants to use check-in data (e.g., from users' smartphones) to create daily histograms of popular visit times of businesses, such as stores or restaurants. This could allow other users to estimate how busy a location or area is during different times of the day, or provide store owners with insights on customer activity. The desired data is often privacy-sensitive, so users may distrust the data collector and be reluctant to share their whereabouts during the course of the day. To enable such use cases in a privacy-preserving way, we follow the local model and sanitize each user's data before it is collected and aggregated into histograms.

Dataset description. We use the publicly available Gowalla dataset from [6]. Gowalla was a location-based social networking website where users could share their locations by checking in. It contains a total of 6,442,890 check-ins with their location and time recorded between Feb. 2009 and Oct. 2010.

4.3.1 Independent analysis of temporal and spatial data. We simulate data collection in the local model by perturbing the time-of-day and location of each check-in independently.

For the periodic times-of-day, we consider all check-ins at the top 100 locations. We follow a sanitization procedure as with the sleep data in Section 4.2.2 and use the VMF and Purkayastha mechanisms on $\mathbb{S}^{1}$, with Clipped (CL) and Wrapped Laplace (WL) as baselines (cf. Sections 3.2, 3.3 and 3.6). Similarly, to sanitize the locations, we take all check-ins from the top 100 users and represent them as unit vectors on $\mathbb{S}^{2}$. We then apply the appropriate VMF and Purkayastha mechanisms, with Polar Laplace (cf. Section 3.6.3) as baseline.

After gathering the perturbed data, we compute the following histograms: a check-in time histogram for each of the 100 locations with one bin for each hour of the day, and a check-in location histogram for each of the top 100 users with $90 \times 180$ bins, one for each pair of subsequent degrees of latitude and longitude. To stabilize the results, we repeat this procedure in every setting for 100 runs.

Error metrics. As measures of error between the sanitized and original histograms, we again use the mean absolute error (MAE), as well as the Earth Mover's Distance (EMD) with a suitable distance matrix: For the distance between two check-in time histogram bins, we use their circular distance in hours. For 2D location histograms with latitude-longitude bins, we use the great-circle distance, i.e. the actual surface distance, between the geographic positions on the sphere corresponding to the bin centers. Unlike the MAE or MSE which look at the error of each histogram bin individually, the EMD so provides a measure of error that is aware of the semantics of the underlying data by considering how far off the target bin is from the original bin when counting a perturbed check-in location.

Results. Figure 7a shows the errors for the check-in time histograms. For large $\epsilon$, both Wrapped and Clipped Laplace as well as Purkayastha show similar errors that are lower than VMF. For medium to small $\epsilon$, our directional mechanisms gain an advantage over WL and CL with Purkayastha generally achieving the lowest errors under directional privacy, whereas VMF wins under pure DP when using the smaller $d_{2}$-sensitivity. In this case, CL performs worst with generally large MAE and EMD since virtually all counts will be in the first or last histogram bin. 


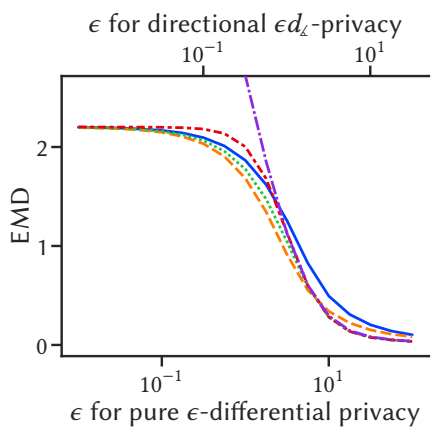

(a) Check-in times

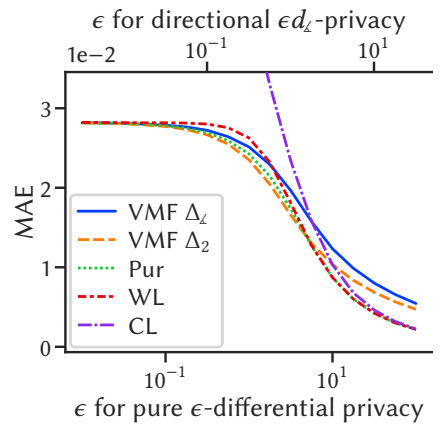

$\epsilon$ for pure $\epsilon$-differential privacy
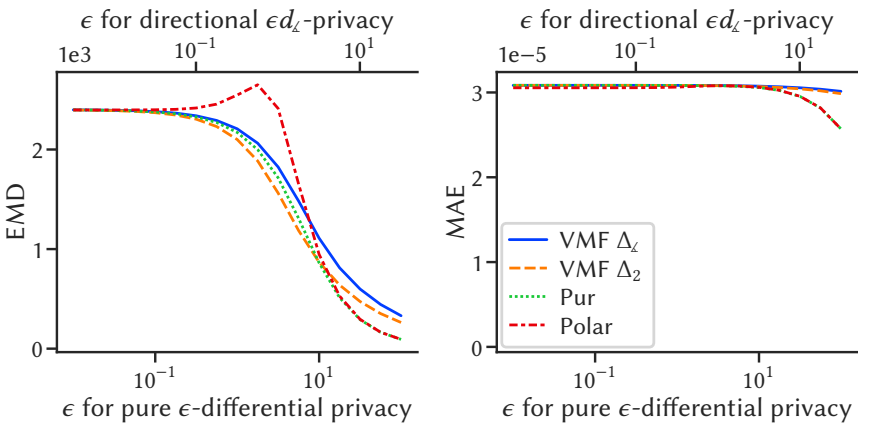

(b) Check-in locations

Figure 7: Comparison of mean absolute error (MAE) and Earth Mover's Distance (EMD) between check-in histograms.

Figure $7 \mathrm{~b}$ shows the errors for the check-in location histograms. In terms of the MAE, VMF is worst while Purkayastha and Polar Laplace are almost indistinguishable. However, if we consider the EMD as metric with spatial awareness, we recognize that the Polar mechanism has a region with increased error for $10^{-1} \lesssim \epsilon \lesssim 10$, corresponding to the "bump" we describe in Section 3.6.3. Thus, in conclusion, the Purkayastha distribution shows the lowest errors for directional privacy, whereas VMF benefits from the reduced $d_{2}$-sensitivity under pure DP.

4.3.2 Location busyness during different times of day. The following experiment constitutes the combined application of directional privacy mechanisms to both spatial and temporal data. Our goal is to derive histograms of check-ins at the top 1000 locations from the Gowalla dataset over different times of day, where we perturb both the check-in times and locations using the Purkayastha mechanism, as well as Wrapped and Polar Laplace as baselines, respectively.

Using differential privacy in the local model is often challenging, since it injects too much noise and hence would make virtually all check-ins probabilistically indistinguishable in our scenario. This is especially problematic for locations, since some areas may be very densely populated with many bars and restaurants-so ideally, we would like to reduce the protection guarantees to reasonably smaller distances. This is an advantage of metric privacy and its variants such as directional privacy, as it allows relaxing the privacy guarantees to a defined protection radius.

Concretely, we sanitize all check-ins at the top 1000 locations by perturbing check-in times on a periodic $24 \mathrm{~h}$ scale with a $\Delta_{\mathrm{t}} \equiv$ 3 hour protection radius, using the 2-dimensional Purkayastha and Wrapped Laplace mechanisms with temporal privacy levels $\ell_{\mathrm{t}} \in\left[10^{-3}, 10^{2}\right]$, and corresponding check-in locations with a $\Delta_{\mathrm{s}} \equiv$ 10 meter protection radius, using the 3 -dimensional Purkayastha and Polar Laplace mechanisms with spatial privacy levels $\ell_{\mathrm{S}} \in$ $\left[10^{-2}, 10^{2}\right]$. We perform 25 repetitions in each setting to obtain stabilized results. For each check-in, we use nearest neighbor search to assign the perturbed check-in coordinates to the nearest location, and aggregate all thusly obtained check-in times at each location into a 24-hour busyness histogram. Similarly, we obtain one daily histogram for each location based on the original, unperturbed data, which we use as reference to compute error metrics for each anonymization run. As in previous experiments, we use the Earth Mover's Distance (EMD) as metric to compare the mechanisms.
Results. Figure 8 shows the EMD over varying temporal and spatial privacy levels $\ell_{\mathrm{t}}$ and $\ell_{\mathrm{s}}$. We clearly see an advantage for the Purkayastha mechanism over the baseline combination of Wrapped and Polar Laplace, which is largest for privacy levels $10^{-1} \lesssim \ell_{\mathrm{t}} \lesssim 1$. We also observe that $\ell_{\mathrm{S}}$ has a less pronounced discriminating effect, as an increase in $\ell_{\mathrm{s}}$ generally reduces the error for both mechanisms, but slightly faster for Purkayastha than Laplace (see also Fig. 12 for a complementary view which shows the spatial and temporal privacy levels $\ell_{\mathrm{t}}$ and $\ell_{\mathrm{s}}$ in the abscissa and columns, respectively). Figure 9 shows exemplary busyness histograms for four selected locations, where the check-in data have been sanitized with privacy levels $\ell_{\mathrm{s}}=\ell_{\mathrm{t}} \approx 0.316$. As we can see, Purkayastha is able to better preserve utility than the baseline mechanisms.

\section{RELATED WORK}

Various DP mechanisms have been proposed for particular types of data: In the context of location data, Andrés et al. [2] introduce the notion of geo-indistinguishability together with the suitable $P L$ mechanism. However, their approach assumes a flat surface instead of a curved one, which restricts its usage to smaller areas where a planar approximation is acceptable. While it is possible to wrap the PL mechanism around the sphere, our experiments in Section 4.3 show that directional mechanisms provide superior utility at the same privacy level when considering global locations.

Data collection in the local model can be dated back to Warner [43], who proposed a method to conduct surveys that allows the respondents to maintain privacy by randomizing their response. The goal is to eliminate evasive answer bias in cases where the individuals may prefer not to reply at all or to reply with incorrect answers to certain sensitive questions. Erlingsson et al. [12] present a modern variant called RAPPOR that privately collects statistics by hashing each user's sensitive value to a Bloom filter [3] and then applying randomized response to each bit in the filter array. Their method applies to discrete values, since hashing only slightly differing floating point values would drastically change their hashes. Kim et al. [23] employ RAPPOR to collect indoor positioning data based on a finite set of preinstalled indoor beacons.

Hay et al. $[16,17]$ evaluate existing DP histogram mechanisms in the central model. Compared to our approach with local sanitization, central DP mechanisms typically offer higher utility, but come at the expense of requiring a trusted data aggregator. 

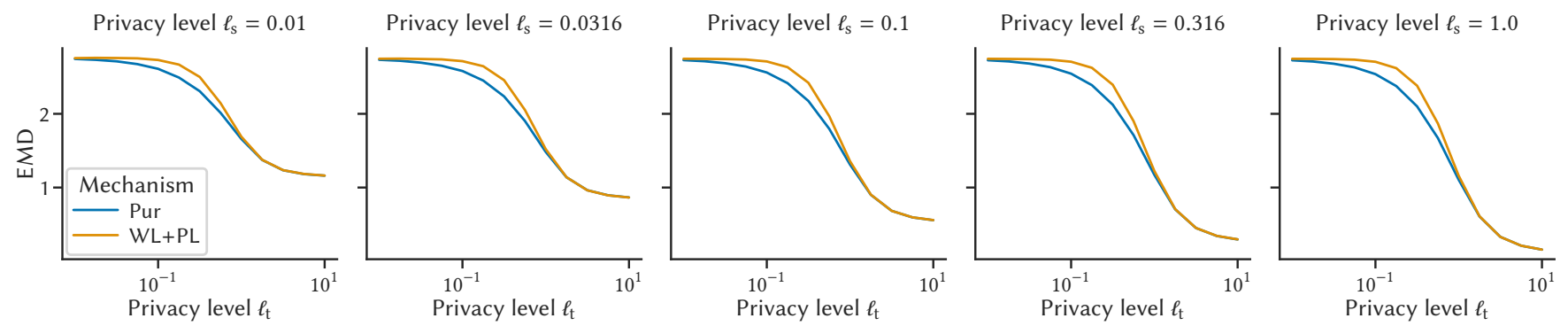

Figure 8: Earth Mover's Distance (EMD) between sanitized and original daily check-in activity histograms over temporal and spatial privacy levels $\ell_{\mathrm{t}}$ (abscissa) and $\ell_{\mathbf{s}}$ (columns) with protection radii $r_{\mathbf{t}} \equiv 3 \mathrm{~h}$ and $r_{\mathbf{s}} \equiv 10 \mathrm{~m}$, respectively.
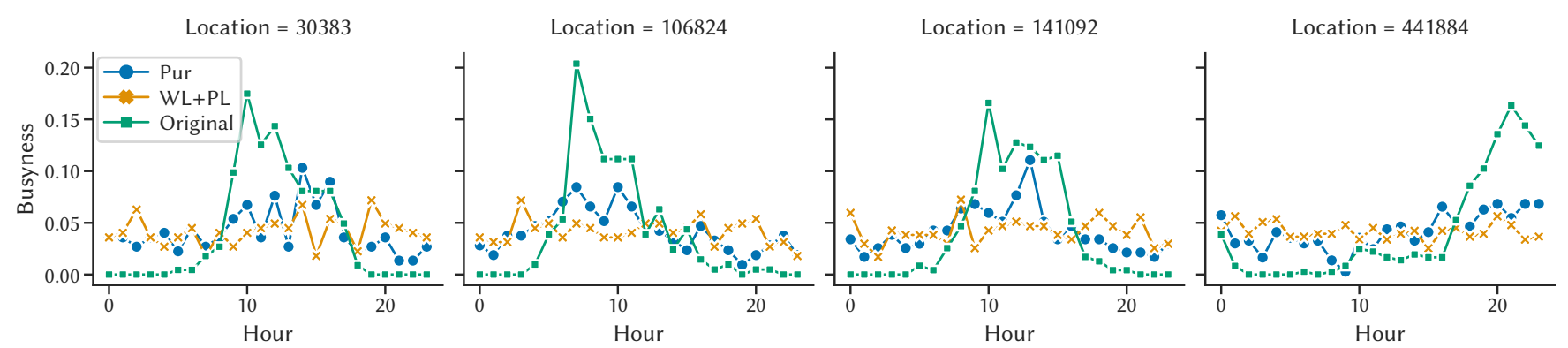

Figure 9: Daily check-in activity for a single sanitization run at exemplary locations, with directional privacy levels $\ell_{\mathbf{s}}=\ell_{\mathbf{t}}=$ $10^{0.5} \approx 0.316$ and protection radii $r_{\mathbf{s}} \equiv 10 \mathbf{m}, r_{\mathbf{t}} \equiv 3 \mathbf{h}$.

Wang et al. [42] propose a matrix-valued variant of the VMF distribution to achieve DP in the context of spectral graph analysis, i.e., computing eigenvalues and -vectors from graph adjacency matrices. While our proof for the VMF mechanism directly works with its probability density, they consider the matrix Bingham$V M F$ distribution with the Exponential Mechanism [31] as auxiliary, which penalizes the privacy guarantee by a factor of 2 .

Kurz and Hanebeck [27] proposed an approximate inversion method for the VMF distribution as alternative to rejection sampling $[38,44]$, which also inspired our Purkayastha sampling scheme. The method relies on the angular $\operatorname{CDF} \operatorname{VMFArc}[\theta \leq \vartheta]$, for which they provide an analytical solution for odd $n$. Unfortunately, for even $n$ the solution contains an infinite series with special functions which we cannot evaluate efficiently. Moreover, as the number of terms grows with $n$, we suspect that the method may only be practical for VMF when the number of dimensions $n$ is both odd and small. On the other hand, we argue that the method is still valuable and practical for the Purkayastha distribution, where our solution for $\operatorname{PurArc}[\theta \leq \vartheta]$ in Eq. (14) provides a closed-form expression with only finitely many terms, regardless of the parity of $n$. Our experiments in Section 4.1 confirm that our approach provides an effective sampling method that works in up to tens of thousands of dimensions, pushing beyond the status quo of 150 dimensions [7].

\section{CONCLUSION}

We have introduced a novel notion of directional privacy for the important class of directional data. To realize this notion, we have suggested the VMF and Purkayastha mechanisms which are based on spherical distributions and intrinsically suit directional data. We have proved that they also conform to the notion of differential privacy, and derived other statistical properties such as expected distances, related densities and cumulative distribution functions. For the Purkayastha distribution, we have proposed a novel sampling algorithm where previously no method was published.

We performed several analyses and experiments on real data to show the applicability of our mechanisms and demonstrate their advantage over standard privacy mechanisms adapted to directional data: Importantly, we observed that the new directional mechanisms typically require fewer data to achieve a certain accuracy. For directional statistics, such as the circular mean, we demonstrated that the local model may achieve higher utility and hence is preferable over the central one, since it also does not require a trusted aggregator. The facilitated use cases include important applications such as privately collecting mobility data in the local model, where the data collector cannot or may not be trusted by the users.

Further work could include finding other applications or domains for which specialized mechanisms yield improved privacy-utility trade-offs, as well as devising more efficient sampling routines especially in high dimensions.

\section{ACKNOWLEDGMENTS}

This work has received funding from the European Union's Horizon 2020 research and innovation programme under grant agreement No. 825333 (MOSAICrOWN).

\section{REFERENCES}

[1] Milton Abramowitz and Irene Stegun. 1972. Handbook of Mathematical Functions with Formulas, Graphs, and Mathematical Tables (10th printing ed.). Applied 
Mathematics, Vol 55. National Bureau of Standards.

[2] M. Andrés, N. Bordenabe, K. Chatzikokolakis, and C. Palamidessi. 2013. Geoindistinguishability: Differential privacy for location-based systems. In $A C M$ SIGSAC conference on Computer \& communications security. ACM, 901-914.

[3] Burton H Bloom. 1970. Space/time trade-offs in hash coding with allowable errors. Commun. ACM 13, 7 (1970), 422-426.

[4] Konstantinos Chatzikokolakis, Miguel E. Andrés, Nicolás Emilio Bordenabe, and Catuscia Palamidessi. 2013. Broadening the Scope of Differential Privacy Using Metrics. In Privacy Enhancing Technologies. Springer, 82-102.

[5] K. Chatzikokolakis and Marco Stronati. 2015. Location Guard: location privacy for the rest of us. https://github.com/chatziko/location-guard

[6] Eunjoon Cho, Seth A Myers, and Jure Leskovec. 2011. Friendship and mobility: user movement in location-based social networks. In Proceedings of the 17th ACM SIGKDD international conference on Knowledge discovery and data mining 1082-1090.

[7] Christine Cutting, Davy Paindaveine, and Thomas Verdebout. 2017. Tests of Concentration for Low-Dimensional and High-Dimensional Directional Data. Springer International Publishing, Cham, 209-227.

[8] Luc Devroye. 2006. Nonuniform random variate generation. Handbooks in operations research and management science 13 (2006), 83-121.

[9] J. C. Duchi, M. I. Jordan, and M. J. Wainwright. 2013. Local Privacy and Statistical Minimax Rates. In 2013 IEEE 54th Annual Symposium on Foundations of Computer Science. 429-438.

[10] C. Dwork, F. McSherry, K. Nissim, and A. Smith. 2006. Calibrating noise to sensitivity in private data analysis. In Theory of Cryptography Conference. Springer, 265-284.

[11] Cynthia Dwork and Aaron Roth. 2014. The algorithmic foundations of differential privacy. Foundations and Trends ${ }^{\circledR}$ in Theoretical Computer Science 9, 3-4 (2014), 211-407.

[12] Úlfar Erlingsson, Vasyl Pihur, and Aleksandra Korolova. 2014. Rappor: Randomized aggregatable privacy-preserving ordinal response. In Proceedings of the 2014 ACM SIGSAC conference on computer and communications security. 1054-1067.

[13] R. Fisher. 1953. Dispersion on a Sphere. Proceedings of the Royal Society A Mathematical, Physical and Engineering Sciences 217, 1130 (1953), 295-305.

[14] National Sleep Foundation. 2015. 2011 Sleep in America Poll - Technology Use and Sleep. 1, 2 (2015), e10. https://doi.org/10.1016/j.sleh.2015.04.010

[15] Izrail Solomonovich Gradshteyn and Iosif Moiseevich Ryzhik. 2014. Table of Integrals, Series, and Products. Academic Press.

[16] Michael Hay, Ashwin Machanavajjhala, Gerome Miklau, Yan Chen, and Dan Zhang. 2016. Principled Evaluation of Differentially Private Algorithms using DPBench. In Proceedings of the 2016 International Conference on Management of Data. $139-154$.

[17] Michael Hay, Ashwin Machanavajjhala, Gerome Miklau, Yan Chen, Dan Zhang, and George Bissias. 2016. Exploring Privacy-Accuracy Tradeoffs using DPComp. In Proceedings of the 2016 International Conference on Management of Data. 21012104

[18] Alex Hern. 2018. Fitness tracking app Strava gives away location of secret US army bases. (2018). https://www.theguardian.com/world/2018/jan/28/fitnesstracking-app-gives-away-location-of-secret-us-army-bases

[19] Pierre Humbert. 1920. Sur les fonctions hypercylindriques. C. R. Acad. Sci., Paris 171 (1920), 490-492.

[20] Pierre Humbert. 1922. IX.-The Confluent Hypergeometric Functions of Two Variables. Proceedings of the Royal Society of Edinburgh 41 (1922), 73-96.

[21] S Rao Jammalamadaka and TJ Kozubowski. 2003. A new family of circular models The wrapped Laplace distributions. Advances and applications in statistics 3, 1 (2003), 77-103.

[22] Fredrik Johansson et al. 2018. mpmath: a Python library for arbitrary-precision floating-point arithmetic (version 1.1.0). http://mpmath.org/

[23] Jong Wook Kim, Dae-Ho Kim, and Beakcheol Jang. 2018. Application of local differential privacy to collection of indoor positioning data. Ieee Access 6 (2018), 4276-4286.

[24] John Krumm. 2009. A survey of computational location privacy. Personal and Ubiquitous Computing 13, 6 (2009), 391-399.

[25] E.E. Kummer. 1836. Über die hypergeometrische Reihe ... . Journal für die reine und angewandte Mathematik 15 (1836), 39-83.

[26] E.E. Kummer. 1836. Über die hypergeometrische Reihe ... . (Fortsetzung). fournal für die reine und angewandte Mathematik 15 (1836), 127-172.

[27] G. Kurz and U. D. Hanebeck. 2015. Stochastic sampling of the hyperspherical von Mises-Fisher distribution without rejection methods. In 2015 Sensor Data Fusion: Trends, Solutions, Applications (SDF). 1-6.

[28] Siu Kwan Lam, Antoine Pitrou, and Stanley Seibert. 2015. Numba: A LLVM-Based Python JIT Compiler. In Proceedings of the Second Workshop on the LLVM Compiler Infrastructure in HPC (Austin, Texas) (LLVM '15). Association for Computing Machinery, New York, NY, USA, Article 7, 6 pages. https://doi.org/10.1145/ 2833157.2833162

[29] Ninghui Li, Min Lyu, Dong Su, and Weining Yang. 2016. Differential privacy: From theory to practice. Synthesis Lectures on Information Security, Privacy, \& Trust (2016).
[30] Kanti V. Mardia and Peter E. Jupp. 2000. Directional statistics. Vol. 494. John Wiley \& Sons.

[31] Frank McSherry and Kunal Talwar. 2007. Mechanism design via differential privacy. In 48th Annual IEEE Symposium on Foundations of Computer Science (FOCS'07). IEEE, 94-103.

[32] Travis E Oliphant. 2006. A guide to NumPy. Vol. 1. Trelgol Publishing USA.

[33] Vincent Primault, Sonia Ben Mokhtar, Cédric Lauradoux, and Lionel Brunie. 2014. Differentially private location privacy in practice. arXiv preprint arXiv:1410.7744 (2014)

[34] Sumitra Purkayastha. 1991. A rotationally symmetric directional distribution: obtained through maximum likelihood characterization. Sankhyā: The Indian Journal of Statistics, Series A (1991), 70-83.

[35] Apostolos Pyrgelis, Carmela Troncoso, and Emiliano De Cristofaro. 2018. Knock Knock, Who's There? Membership Inference on Aggregate Location Data. In 25th Annual Network and Distributed System Security Symposium, NDSS 2018, San Diego, California, USA, February 18-21, 2018. The Internet Society. http://wp.internetsociety.org/ndss/wp-content/uploads/sites/25/2018/02/ ndss 2018 05B-5_Pyrgelis_paper.pdf

[36] John G Saw. 1978. A family of distributions on the $\mathrm{m}$-sphere and some hypothesis tests. Biometrika 65, 1 (1978), 69-73.

[37] Stuart A. Thompson and Charlie Warzel. 2019. Opinion | Twelve Million Phones, One Dataset, Zero Privacy. (2019). https://www.nytimes.com/interactive/2019/ 12/19/opinion/location-tracking-cell-phone.html

[38] Gary Ulrich. 1984. Computer Generation of Distributions on the M-Sphere. fournal of the Royal Statistical Society: Series C (Applied Statistics) 33, 2 (1984), 158-163.

[39] Pauli Virtanen, Ralf Gommers, Travis E Oliphant, Matt Haberland, Tyler Reddy, David Cournapeau, Evgeni Burovski, Pearu Peterson, Warren Weckesser, Jonathan Bright, et al. 2020. SciPy 1.0: fundamental algorithms for scientific computing in Python. Nature Methods (2020), 1-12.

[40] Richard von Mises. 1918. Über die "Ganzzahligkeit" der Atomgewicht und verwandte Fragen. Physikalische Zeitschrift 19 (1918), 490-500.

[41] Stéfan van der Walt, S Chris Colbert, and Gael Varoquaux. 2011. The NumPy array: a structure for efficient numerical computation. Computing in Science \& Engineering 13, 2 (2011), 22-30.

[42] Yue Wang, Xintao Wu, and Leting Wu. 2013. Differential privacy preserving spectral graph analysis. In Pacific-Asia Conference on Knowledge Discovery and Data Mining. Springer, 329-340.

[43] Stanley L Warner. 1965. Randomized response: A survey technique for eliminating evasive answer bias. 7. Amer. Statist. Assoc. 60, 309 (1965), 63-69.

[44] Andrew T.A Wood. 1994. Simulation of the von Mises Fisher distribution. Communications in Statistics - Simulation and Computation 23, 1 (1994), 157-164.

\section{A SUPPLEMENTARY MATERIAL}

\section{A.1 Special functions and notation}

Directional distributions (as considered in Sections 2.2 and 3) often depend on certain special functions and their properties. We hereafter provide an overview of those functions required in this paper, and briefly summarize their key properties and relations. Further details can be found in $[1,15]$, for example.

\section{A.1.1 Gamma and Beta functions.}

Definition 25. The Gamma function (or Euler integral of the second kind) is defined for $z \in \mathbb{C}$ with real part $\mathfrak{R}(z)>0$ as

$$
\Gamma(z)=\int_{0}^{\infty} t^{z-1} e^{-t} \mathrm{~d} t .
$$

Important special values are $\Gamma(1)=1$ and $\Gamma\left(\frac{1}{2}\right)=\sqrt{\pi}$. It has a functional relation $\Gamma(z+1)=z \Gamma(z)$, so $\Gamma(n+1)=n$ ! for $n \in$ $\mathbb{N}$. Therefore, $\Gamma$ provides an extension of the factorial to complex numbers.

Definition 26. The Pochhammer symbol (or rising factorial) with $k$ factors is defined as

$$
(a)_{k}:=\frac{\Gamma(a+k)}{\Gamma(a)}=a(a+1) \cdots(a+k-1)
$$

with the convention that $(a)_{0}=1$. 
Definition 27. The Beta function (or Euler integral of the first kind) is defined for $x, y \in \mathbb{C}$ with $\mathfrak{R}(x), \mathfrak{R}(y)>0$ as

$$
\mathrm{B}(x, y)=\int_{0}^{1} t^{x-1}(1-t)^{y-1} \mathrm{~d} t .
$$

It is symmetric in its arguments. Particular relations are:

$$
\begin{aligned}
\mathrm{B}(x, y) & =\frac{\Gamma(x) \Gamma(y)}{\Gamma(x+y)} \\
\mathrm{B}\left(\frac{1}{2}, x\right) & =2^{2 x-1} \mathrm{~B}(x, x)
\end{aligned}
$$

\section{A.1.2 Confluent hypergeometric series.}

Definition 28. Kummer's (confluent hypergeometric) function, denoted by $M(\alpha ; \gamma ; z)$ or ${ }_{1} F_{1}(\alpha ; \gamma ; z)$, is a confluent hypergeometric series given by Kummer $[25,26]$ as

$$
M(\alpha ; \gamma ; z)={ }_{1} F_{1}(\alpha ; \gamma ; z)=\sum_{k=0}^{\infty} \frac{(\alpha)_{k}}{(\gamma)_{k}} \frac{z^{k}}{k !} .
$$

For $\mathfrak{R} \gamma>\mathfrak{R} \alpha>0$, it has an integral representation

$$
M(\alpha ; \gamma ; z)=\frac{\Gamma(\gamma)}{\Gamma(\alpha) \Gamma(\gamma-\alpha)} \int_{0}^{1} t^{\alpha-1}(1-t)^{\gamma-\alpha-1} e^{z t} \mathrm{~d} t .
$$

Definition 29. The modified Bessel function of the first kind of order $v \in \mathbb{R}$ is given by the series

$$
I_{v}(z)=\sum_{k=0}^{\infty} \frac{1}{k ! \Gamma(k+v+1)}\left(\frac{x}{2}\right)^{2 k+v} .
$$

For $\mathfrak{R}(v)>-\frac{1}{2}$, it can be represented as integral, e.g.

$$
I_{v}(z)=\frac{\left(\frac{z}{2}\right)^{v}}{\Gamma\left(v+\frac{1}{2}\right) \Gamma\left(\frac{1}{2}\right)} \int_{-1}^{1}\left(1-t^{2}\right)^{v-\frac{1}{2}} e^{ \pm z t} \mathrm{~d} t .
$$

We can express $I_{v}(z)$ in terms of Kummer's function:

$$
I_{v}(z)=\frac{e^{-z}}{\Gamma(v+1)}\left(\frac{z}{2}\right)^{v} M\left(v+\frac{1}{2}, 2 v+1 ; 2 z\right)
$$

Humbert series. Humbert $[19,20]$ introduced a set of seven hypergeometric double series that generalize Kummer's confluent hypergeometric series to two variables. One example we use is

Definition 30. The Humbert series $\Phi_{1}$ is defined for $|x|<1$ by a confluent hypergeometric series of two variables

$$
\Phi_{1}(\alpha, \beta, \gamma ; x, y)=\sum_{m, n=0}^{\infty} \frac{(\alpha)_{m+n}(\beta)_{m}}{(\gamma)_{m+n}} \frac{x^{m} y^{n}}{m ! n !} .
$$

For $\mathfrak{R} \gamma>\mathfrak{R} \alpha>0$, it has an integral representation

$$
\frac{\Gamma(\gamma)}{\Gamma(\alpha) \Gamma(\gamma-\alpha)} \int_{0}^{1} t^{\alpha-1}(1-t)^{\gamma-\alpha-1}(1-x t)^{-\beta} e^{y t} \mathrm{~d} t .
$$

\section{A.2 Proofs omitted from Section 2}

Proof of Lemma 8. Since $\mathrm{P}[\mathrm{x}]=\overline{\mathrm{P}}[t]$ for $t=\boldsymbol{\mu}^{\top} \mathrm{x}$ and $t$ is fixed in the integral, we can pull out the kernel function and obtain

$$
\operatorname{PMix}[t]=\int_{\mathbf{x}: \mu^{\top} \mathbf{x}=t} \mathrm{P}[\mathbf{x}] \mathrm{dx}=\overline{\mathrm{P}}[t] \int_{\mathbf{x}: \boldsymbol{\mu}^{\top} \mathbf{x}=t} 1 \mathrm{~d} \mathbf{x} .
$$

To evaluate the remaining integral, first note that the points $\mathbf{x} \in$ $\mathbb{S}^{n-1}$ with $\boldsymbol{\mu}^{\top} \mathbf{x}=t$ form an $(n-2)$-dimensional subsphere centered at $t \boldsymbol{\mu}$ with radius $\sqrt{1-t^{2}}$ that is orthogonal to $\mu$. By Fact 6 , its $(n-2)$-dimensional surface area is

$$
\operatorname{vol}\left(\sqrt{1-t^{2}} \mathbb{S}^{n-2}\right)=S_{n-2} \cdot\left(1-t^{2}\right)^{\frac{n-2}{2}}
$$

The angle between the modal axis and the subsphere in terms of $t$ is $\arccos (t)$; with respect to the differential $\mathrm{d} t$, the subsphere's width on the surface along $\boldsymbol{\mu}$ hence is $|\mathrm{d} / \mathrm{d} t \arccos (t)|=1 / \sqrt{1-t^{2}}$. Overall, the surface element amounts to $S_{n-2} \cdot\left(1-t^{2}\right)^{(n-3) / 2}$, so we can express the mixture density in terms of the kernel function $\bar{P}$ as

$$
\operatorname{PMix}[t]=S_{n-2} \cdot\left(1-t^{2}\right)^{\frac{n-3}{2}} \cdot \overline{\mathrm{P}}[t] .
$$

\section{A.3 Proofs omitted from Section 3}

\section{A.3.1 Proofs related to the VMF distribution.}

Proof of Theorem 15. Let $x, y \in \mathbb{S}^{n-1}$ be any fixed unit vectors, and take any fixed set $\mathbf{Z} \subseteq \mathbb{S}^{n-1}$. For any $\mathbf{z} \in \mathbf{Z}$, we have

$$
\begin{aligned}
\frac{\operatorname{VMF}(x, \epsilon)[\mathrm{z}]}{\operatorname{VMF}(\boldsymbol{y}, \epsilon)[\mathrm{z}]} & =\frac{C_{\mathrm{VMF}} \cdot \exp \left(\epsilon \cdot \boldsymbol{x}^{\top} \mathbf{z}\right)}{C_{\mathrm{VMF}} \cdot \exp \left(\epsilon \cdot \boldsymbol{y}^{\top} \mathbf{z}\right)} \\
& =\exp \left(\epsilon \cdot(\boldsymbol{x}-\boldsymbol{y})^{\top} \mathbf{z}\right) \\
& \leq \exp \left(\epsilon \cdot\|\boldsymbol{x}-\boldsymbol{y}\|_{2} \cdot\|\mathbf{z}\|_{2}\right) \\
& =\exp \left(\epsilon \cdot d_{2}(\boldsymbol{x}, \boldsymbol{y})\right) .
\end{aligned}
$$

First, the normalization constants cancel out, and we can combine the exponents; the inequality is the Cauchy-Schwarz inequality; finally, note that $\|\mathbf{z}\|_{2}=1$. By integrating over $\mathbf{z} \in \mathbf{Z}$, we achieve $\epsilon d_{2}$-privacy.

Proof of Theorem 17. Because of the rotational symmetry, we can write $d_{2}(\boldsymbol{x}, \boldsymbol{\mu})=\sqrt{2} \sqrt{1-t}$ in terms of the mixture variable $t$. Therefore,

$$
\begin{aligned}
\underset{\mathbf{x} \sim \operatorname{VMF}}{\mathbb{E}}\left[d_{2}(\mathbf{x}, \boldsymbol{\mu})\right] & =\underset{t \sim \operatorname{VMFMix}}{\mathbb{E}}[\sqrt{2} \sqrt{1-t}] \\
& =\int_{-1}^{1} \sqrt{2} \sqrt{1-t} \cdot \operatorname{VMFMix}[t] \mathrm{d} t \\
& =C_{\mathrm{VMF}}^{\prime} \sqrt{2} \int_{-1}^{1} e^{\kappa t} \sqrt{1-t}\left(1-t^{2}\right)^{\frac{n-3}{2}} \mathrm{~d} t \\
& =C_{\mathrm{VMF}}^{\prime} \sqrt{2} \int_{-1}^{1} e^{\kappa t}(1-t)^{\frac{n-2}{2}}(1+t)^{\frac{n-3}{2}} \mathrm{~d} t
\end{aligned}
$$

Changing variables $t \mapsto \frac{t+1}{2}$ results in

$$
=C_{\mathrm{VMF}}^{\prime} 2^{n-1} e^{-\kappa} \int_{0}^{1} e^{2 \kappa t}(1-t)^{\frac{n-2}{2}} t^{\frac{n-3}{2}} \mathrm{~d} t,
$$

where we can express the integral as a Kummer function by Eq. (25), and then simplify with the normalization constant:

$$
\begin{aligned}
& =C_{\mathrm{VMF}}^{\prime} 2^{n-1} e^{-\kappa} \mathrm{B}\left(\frac{n-1}{2}, \frac{n}{2}\right) M\left(\frac{n-1}{2} ; n-\frac{1}{2} ; 2 \kappa\right) \\
& =2^{n-1} \frac{\mathrm{B}\left(\frac{n-1}{2}, \frac{n}{2}\right)}{\mathrm{B}\left(\frac{1}{2}, \frac{n-1}{2}\right)} \frac{M\left(\frac{n-1}{2} ; n-\frac{1}{2} ; 2 \kappa\right)}{M\left(\frac{n-1}{2} ; n-1 ; 2 \kappa\right)} \\
& =\frac{\mathrm{B}\left(\frac{1}{2}, \frac{n}{2}\right)}{\mathrm{B}\left(\frac{1}{2}, n-\frac{1}{2}\right)} \frac{M\left(\frac{n-1}{2} ; n-\frac{1}{2} ; 2 \kappa\right)}{M\left(\frac{n-1}{2} ; n-1 ; 2 \kappa\right)} .
\end{aligned}
$$

The last step follows by rewriting the fraction of Beta functions where we apply Eq. (23) to expand the numerator to $2^{1-n} \cdot \mathrm{B}\left(\frac{1}{2}, \frac{n}{2}\right)$. 
Proof of Theorem 18. With $\alpha:=\frac{n-1}{2}$ and $x:=\frac{T+1}{2}$, we obtain

$$
\begin{aligned}
\operatorname{VMFMix}(n, \kappa)[t \leq T] & =\int_{-1}^{T} \operatorname{VMFMix}(n, \kappa)[t] \mathrm{d} t \\
& =C_{\mathrm{VMF}}^{\prime} \int_{-1}^{T}\left(1-t^{2}\right)^{\frac{n-3}{2}} e^{\kappa t} \mathrm{~d} t .
\end{aligned}
$$

Changing variables $t \mapsto \frac{t+1}{T+1}$ yields an integral that we can express as Humbert series according to Eq. (30), so we get

$$
\begin{aligned}
= & C_{\mathrm{VMF}}^{\prime} 2^{n-2} x^{\frac{n-1}{2}} e^{-\kappa} \\
& \cdot \int_{0}^{x} e^{2 \kappa x t} t^{\frac{n-3}{2}}(1-x t)^{\frac{n-3}{2}} \mathrm{~d} t \\
= & \frac{x^{\alpha}}{\alpha} \frac{\Phi_{1}(\alpha, 1-\alpha, 1+\alpha ; x, 2 \kappa x)}{\mathrm{B}(\alpha, \alpha) M(\alpha, 2 \alpha, 2 \kappa)} .
\end{aligned}
$$

\section{A.3.2 Proofs related to the Purkayastha distribution.}

Proof of Theorem 19. Let $\boldsymbol{x}, \boldsymbol{y} \in \mathbb{S}^{n-1}$ be any fixed unit vectors, and take any fixed set $\mathbf{Z} \subseteq \mathbb{S}^{n-1}$. For any $\mathbf{z} \in \mathbf{Z}$, we have

$$
\begin{aligned}
\frac{\operatorname{Pur}(\boldsymbol{x}, \epsilon)[\mathbf{z}]}{\operatorname{Pur}(\boldsymbol{y}, \epsilon)[\mathbf{z}]} & =\frac{C_{\text {Pur }} \cdot \exp \left(-\epsilon \cdot \arccos \left(\boldsymbol{x}^{\top} \mathbf{z}\right)\right)}{C_{\text {Pur }} \cdot \exp \left(-\epsilon \cdot \arccos \left(\boldsymbol{y}^{\top} \mathbf{z}\right)\right)} \\
& =\exp \left(\epsilon \cdot \arccos \left(\boldsymbol{y}^{\top} \mathbf{z}\right)-\arccos \left(x^{\top} \mathbf{z}\right)\right) \\
& \leq \exp \left(\epsilon \cdot \arccos \left(\boldsymbol{x}^{\top} \boldsymbol{y}\right)\right) \\
& =\exp \left(\epsilon \cdot d_{\iota}(\boldsymbol{x}, \boldsymbol{y})\right) .
\end{aligned}
$$

First, the normalization constants cancel out, and we can combine the exponents; next, we apply the triangle inequality for the angular (arccos) distance. By integrating over $\mathbf{z} \in \mathbf{Z}$, we obtain $\epsilon d_{L}$-privacy.

Proof of Lemma 21. Note that for any $\ell \in \mathbb{Z}$,

$$
\mathcal{T}_{k}(\ell \pi)= \begin{cases}0 & \text { for } k<m, \\ a & \text { for } k=m \text { and even } n, \\ (-1)^{\ell+1} & \text { for } k=m \text { and odd } n,\end{cases}
$$

Therefore,

$$
\begin{aligned}
E_{n, a}(\ell \pi) & =e^{a \ell \pi} C_{m} \mathcal{T}_{m}(\ell \pi) \\
& =e^{a \ell \pi} C_{m} \begin{cases}a & \text { if } n \text { is even, } \\
(-1)^{\ell+1} & \text { if } n \text { is odd, }\end{cases}
\end{aligned}
$$

and we obtain as special case the normalization constant

$$
F_{n, a}(\pi)=C_{m} \begin{cases}a\left(e^{a \pi}-1\right) & \text { if } n \text { is even, } \\ \left(e^{a \pi}+1\right) & \text { if } n \text { is odd. }\end{cases}
$$

Proof of Theorem 22. The surface distance $\theta=d_{\llcorner}(\mathbf{x}, \boldsymbol{\mu})$ follows the angular distribution $\theta \sim \operatorname{PurArc}(n, \kappa)$. Therefore, we have

$$
\begin{aligned}
\underset{\mathbf{x} \sim \operatorname{Pur}}{\mathbb{E}}\left[d_{L}(\mathbf{x}, \boldsymbol{\mu})\right] & =\underset{\theta \sim \operatorname{PurArc}}{\mathbb{E}}[\theta] \\
& =C_{\text {Pur }}^{\prime} \int_{0}^{\pi} \theta e^{-\kappa \theta} \sin ^{n-2}(\theta) \mathrm{d} \theta .
\end{aligned}
$$

Since $\theta e^{-\kappa \theta}=-\frac{\partial}{\partial \kappa} e^{-\kappa \theta}$, we can apply Leibniz' rule and Lemma 21:

$$
\begin{aligned}
& =-C_{\text {Pur }}^{\prime} \int_{0}^{\pi} \frac{\partial}{\partial \kappa} e^{-\kappa \theta} \sin ^{n-2}(\theta) \mathrm{d} \theta \\
& =-C_{\text {Pur }}^{\prime} \frac{\partial}{\partial \kappa} \int_{0}^{\pi} e^{-\kappa \theta} \sin ^{n-2}(\theta) \mathrm{d} \theta \\
& =\frac{\frac{\partial}{\partial \kappa} F_{n-2,-\kappa}(\pi)}{-F_{n-2,-\kappa}(\pi)} .
\end{aligned}
$$

The result follows from applying the generalized product rule.

\section{A.3.3 Proofs related to circular baselines.}

Proof of Theorem 24. We compute the exact difference of the expected values and apply the inequality $e^{x} \geq 1+x$ :

$$
\begin{aligned}
\underset{\theta \sim \mathrm{WLArc}}{\mathbb{E}}[\theta]-\underset{\theta \sim \operatorname{PurArc}}{\mathbb{E}}[\theta] & =\frac{\pi}{e^{\kappa \pi}-1}-\frac{2}{\kappa\left(e^{\kappa \pi}+1\right)} \\
& =\frac{e^{\kappa \pi}(\kappa \pi-2)+\kappa \pi+2}{\kappa\left(e^{2 \kappa \pi}-1\right)} \\
& \geq \frac{(1+\kappa \pi)(\kappa \pi-2)+\kappa \pi+2}{\kappa\left(e^{2 \kappa \pi}-1\right)} \\
& =\frac{\kappa \pi^{2}}{e^{2 \kappa \pi}-1}>0 .
\end{aligned}
$$

The limits are trivial for $\kappa \rightarrow \infty$. For $\kappa \rightarrow 0$, they follow from l'Hôpital's rule.

\section{B ADDITIONAL EXPERIMENTS AND RESULTS}

This section provides supplementary experiments that we left out in the main part. Firstly, we verify the theoretical properties of directional distributions we derived in Section 3. Lastly, we provide complementary plots for the error induced in the busyness histograms in Section 4.3 .2 by the spatial and temporal anonymization of the check-in data.

\section{B.1 Empirical verification through simulation}

The following experiments aim at verifying the analytic formula for the expected distances and CDFs of the VMF and Purkayastha distribution we derived in Section 3. We furthermore use them to compare the corresponding mechanisms' utility at a given privacy level.

B.1.1 Expected distances. First, we want to check the correctness of the derived analytical formula Eq. (6) for the expected Euclidean distance $\mathbb{E}_{t \sim V M F M i x}[\sqrt{2} \sqrt{1-t}]$ of the VMF distribution as well as Eq. (13) for the surface distance $\mathbb{E}_{\theta \sim \operatorname{PurArc}}[\theta]$ of the Purkayastha distribution. To that end, we draw 1 million samples from each distribution VMFMix $(n, \kappa)$ and $\operatorname{PurMix}(n, \kappa)$, and compute the empirical means of the corresponding Euclidean and angular distances. We chose $n \in\{2,3,25,50,100,500\}$ and $\kappa \in\left\{10^{k} \mid-3 \leq k \leq 3\right\}$. We compare the thusly obtained empirical distances against the results given by the analytical formula we implemented in Python.

Results. As we can observe in Fig. 10, our analytical solution (marked by X's) precisely predicts the empirical distances, even in higher dimensions such as $n=500$. Moreover, given the same concentration parameter $\kappa$, the Purkayastha distribution consistently 

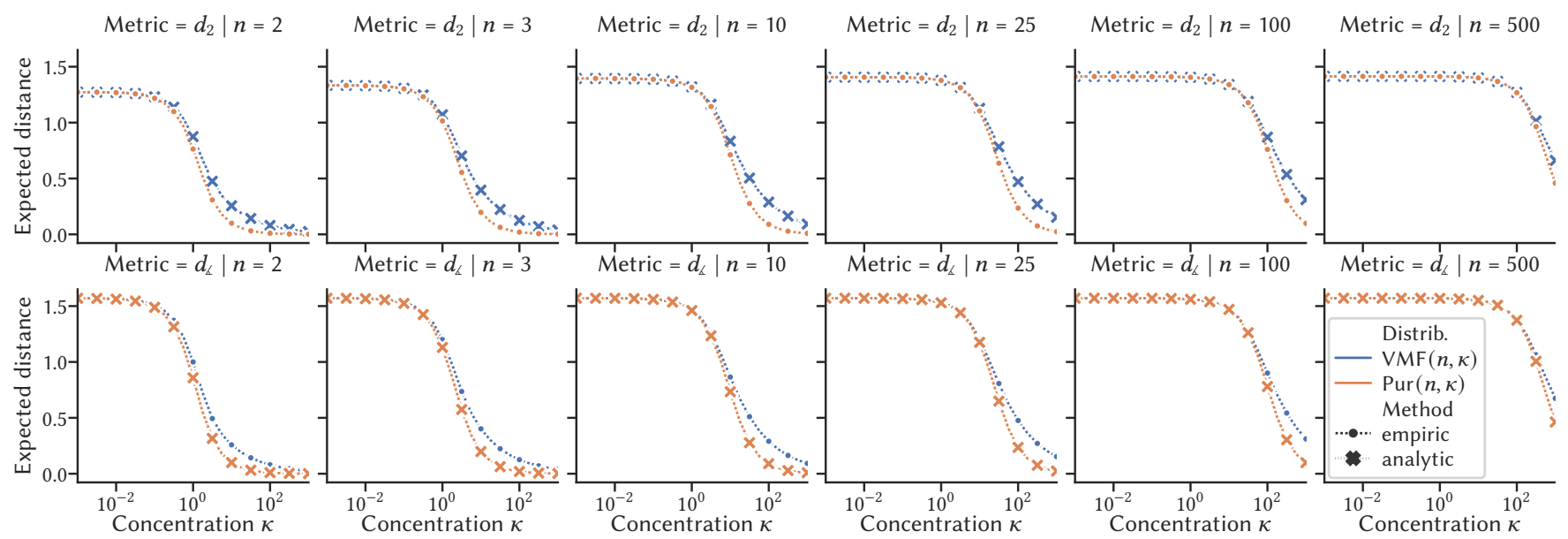

Figure 10: Expected $d_{2}$ and $d_{\measuredangle}$ distances for VMF and Purkayastha distributions in various settings. For comparison, we obtained empirical averages (dotted lines) from 1M samples of each distribution and analytic solutions (X's) from Eqs. (6) and (13).

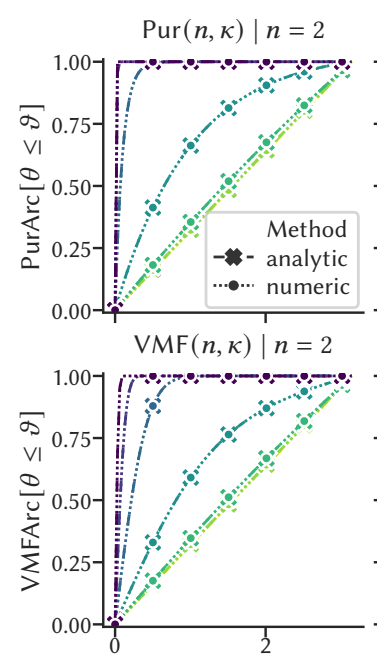

Angle upper bound $\vartheta$

\section{$\operatorname{Pur}(n, \kappa) \mid n=3$}
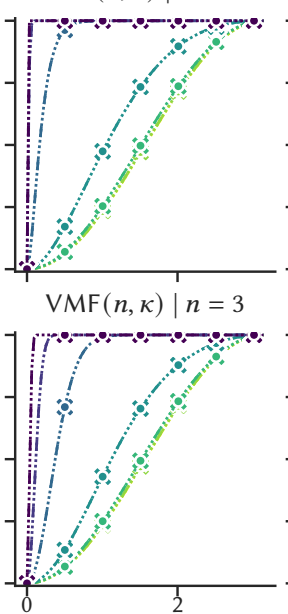

Angle upper bound $\vartheta$
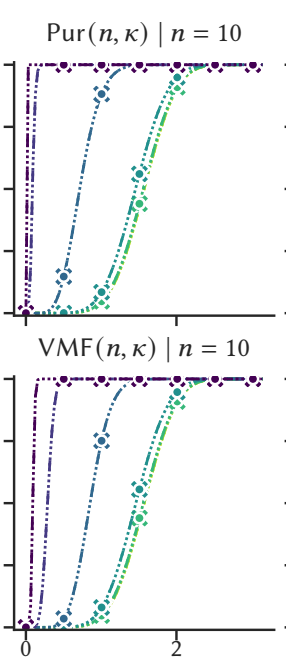

Angle upper bound $\vartheta$

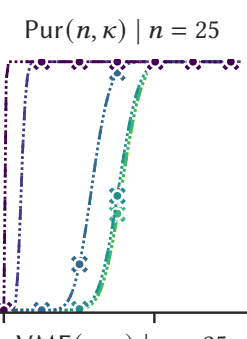

$\operatorname{VMF}(n, \kappa) \mid n=25$

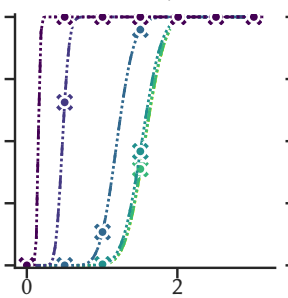

Angle upper bound $\vartheta$
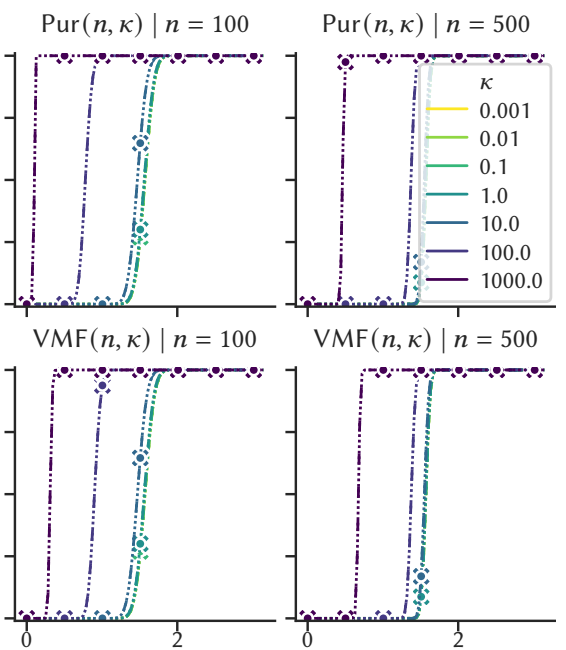

Angle upper bound $\vartheta$

Angle upper bound $\vartheta$

Figure 11: Angular CDFs, obtained via numerical integration (dotted) of the PDFs and analytically (X's) via Eqs. (7) and (14).

has lower expected distance (or error) which indicates that it has a generally more favorable privacy-utility trade-off.

B.1.2 Cumulative distribution functions. Secondly, we want to verify our formula for the CDFs VMFMix $[t \leq T]$ given in Eq. (7) as well as $\operatorname{PurArc}[\theta \leq \vartheta]$ given in Eq. (14). As reference values, we numerically approximate the integrals over the probability density functions using the quadrature routines provided by scipy.

Results. The results are presented in Fig. 11, where we vary $\kappa$ and $n$ as in the previous section. Note that while we derived a solution for the mixture CDF for the VMF distribution, we show the angular $\mathrm{CDFs}$ for both distributions by means of the transformation $\operatorname{VMFArc}[\theta \leq \vartheta]=1-\operatorname{VMFMix}[t \leq \cos (\vartheta)]$ for better comparability and clarity of presentation. Again, we can observe that our analytical solutions (marked by X's) accurately predict the numerical approximations (dotted lines). While the CDFs of both distributions look similar for small $\kappa$, PurArc $[\theta \leq \vartheta]$ grows more rapidly than $\operatorname{VMFArc}[\theta \leq \vartheta]$ as $\vartheta \rightarrow \pi$ for larger $\kappa$, indicating a higher concentration near the mode and hence a better privacy-utility trade-off for directional privacy.

\section{B.2 Additional plots for busyness experiments}

For a different perspective than Fig. 8, Fig. 12 shows the averaged EMD between daily busyness histograms with selected temporal privacy levels $\ell_{\mathrm{t}}$ in the columns and continuous spatial privacy level $\ell_{\mathrm{S}}$ in the abscissa. 


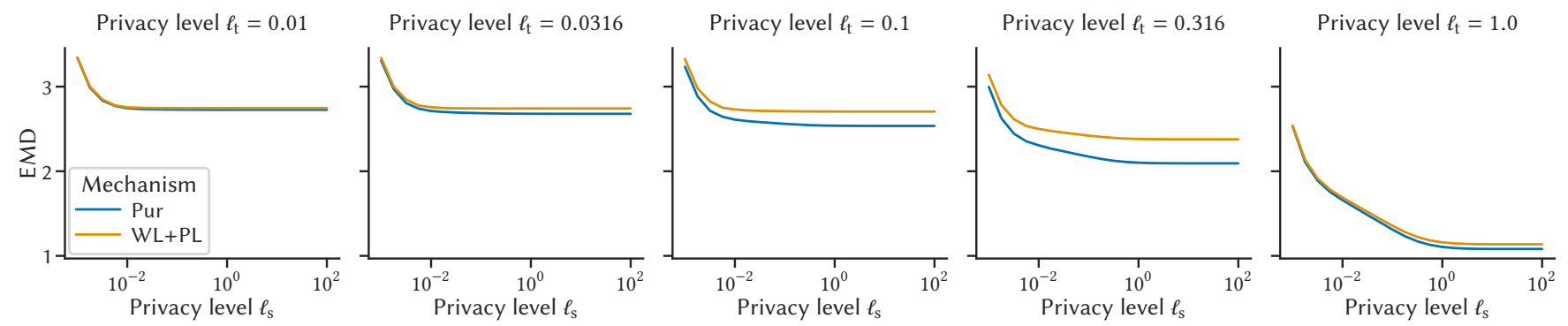

Figure 12: Earth Movers Distance (EMD) over spatial and temporal privacy levels $\ell_{\mathrm{s}}$ (abscissa) and $\ell_{\mathrm{t}}$ (columns) with protection radii $r_{\mathbf{t}} \equiv 3 \mathbf{h}$ and $r_{\mathbf{s}} \equiv 10 \mathrm{~m}$, respectively. 